Ti me Course Effect of R-Al pha- Li poi c Aci d on Cel I ul ar Met abol omi cs in Cul t ur ed Hepat oma Cel Is

\begin{tabular}{|l|l|}
\hline 著者 & $\begin{array}{l}\text { I kut a Naoko, Chi kanto Kei ta, Asano Yuya, } \\
\text { Yasui Yoshi aki, Yokokawa Har uka, Ter ao Kei j i, } \\
\text { Ri mbach Ger al d, Nat sugo Sei i chi }\end{array}$ \\
\hline $\begin{array}{l}\text { j our nal or } \\
\text { publ i cat i on ti tl e }\end{array}$ & Journal of Medi ci nal Food \\
\hline vol une & 20 \\
\hline nunber & 3 \\
\hline page range & $211-222$ \\
\hline year & $2017-03-01$ \\
\hline URL & ht t p: //hdl . handl e. net /2297/48250 \\
\hline
\end{tabular}


Title

\title{
Time-course Effect of R-Alpha-Lipoic Acid on Cellular Metabolomics in Cultured Hepatoma Cells
}

\author{
Naoko Ikuta ${ }^{1}$, Keita Chikamoto ${ }^{2}$, Yuya Asano ${ }^{2}$, Yoshiaki Yasui ${ }^{2}$, Haruka Yokokawa ${ }^{2}$, Keiji Terao $^{3}$, \\ Gerald Rimbach $^{4}$ and Seiichi Matsugo ${ }^{2, *}$ \\ 1 Graduate School of Medicine, Kobe University, Kobe 650-0017, Japan; E-Mail: naoko.ikuta@people.kobe-u.ac.jp \\ (N.I.) \\ 2 College of Science and Engineering, Kanazawa University, Kanazawa 920-1192, Japan; E-Mails: \\ k.chikamoto@gmail.com (K.C.); asano-fd3s@stu.kanazawa-u.ac.jp (Y.A.); x_xve1b@yahoo.co.jp (Y.Y.); \\ haruside8133@gmail.com (H.Y.); s-matsugoh@se.kanazawa-u.ac.jp (S.M.) \\ 3 CycloChem Bio Co., Ltd., Kobe 650-0047, Japan; E-Mail: keiji.terao@cyclochem.com (K.T.) \\ 4 Institute of Human Nutrition and Food Science, University of Kiel, Hermann-Rodewald-Straße 6, 24118 Kiel, \\ Germany; E-Mail: rimbach@foodsci.uni-kiel.de (G.R.) \\ * Correspondence: s-matsugoh@se.kanazawa-u.ac.jp; Tel.: +81-76-264-6219 ; Fax: +81-76-234-4829.
}


Abstract: Alpha-lipoic acid (LA) is a powerful antioxidant. LA has two enantiomers, R(+)-LA (R-LA) and S(-)-LA (S-LA). Of these, R-LA is naturally occurring and an essential cofactor in energy metabolism. R-LA treatment has been reported to affect glucose metabolism in rat heptatoma cells. This study analyzed the time-course of metabolite levels in LA-treated cultured H4IIEC3 rat hepatoma cells, including a specific evaluation of the effect of R-LA and the enantioselectivity of LA. Principal component analysis showed that this experiment was well designed to observe enantioselectivity. R-LA treatment was found to inhibit the glycolysis and Thr-Gly-Ser pathways, as well as lactic acid production, leading to the inhibition of gluconeogenesis in starved H4IIEC3 cells. Present data may provide mechanistic insight by which R-LA induces apoptosis in hepatoma cells.

Keywords: lipoic acid; metabolomics; enantioselectivity; glyconeogenesis; H4IIEC3; hepatoma cells; amino acids;

\section{Introduction}

Alpha-lipoic acid (LA) is a powerful antioxidant, leading to its use in pharmaceuticals and nutraceuticals. The $\mathrm{C}_{6}$ carbon of LA exhibits a chiral center (Appendix figure A1(a), (b)), resulting in two enantiomers, $\mathrm{R}(+)$-LA (R-LA) and S(-)-LA (S-LA). R-LA occurs naturally and is a cofactor for mitochondrial enzymes, such as pyruvate, $\alpha$-ketoglutarate, and branched-chain $\alpha$-ketoacid dehydrogenases ${ }^{1}$, thereby playing an important role in energy metabolism. Pure R-LA is sensitive to physical stimuli such as light and heat. Recently it has been reported that the stabilized R-LA affects energy expenditure in laboratory mice.,3 Nevertheless, commercially available LA is mainly a racemate.

Many studies on LA have been done in cells ${ }^{4,5}$ and animals ${ }^{2}$ because of its antioxidant properties, and particularly the prevention and/or treatment of diabetic complications. ${ }^{6}$ LA has been shown to stimulate glucose uptake, via both GLUT4 translocation and GLUT4 activation, in 3T3-L1 adipocytes and L6 myotubes $^{7}$. In both cell types, R-LA was more effective than S-LA or the racemic mixture and was comparable with insulin-induced. ${ }^{8}$ In tumor cell lines, however, R-LA can reduce cell viability/proliferation, uptake of [18F]-FDG and lactate production and increase apoptosis. ${ }^{9}$ LA induces apoptosis by increasing mitochondrial respiration, along with the concomitant generation of superoxide anion free radicals $\left(\mathrm{O}_{2}{ }^{--}\right)$, in human colon cancer ${ }^{10}$, lung cancer $^{11}$ and hepatoma ${ }^{12}$ cells. Exposure of primary cultured rat hepatocytes to therapeutically relevant concentrations of R-LA for 3 hours was found to increase pyruvate oxidation, apparently by activation of the PDH complex, and to decrease gluconeogenesis and free fatty acid (FFA) oxidation. ${ }^{13}$ Another study reported that the anti-apoptotic effect of R-LA was due to its activation of the insulin receptor/PI3-kinase/Akt pathway. ${ }^{14}$ In that study, R-LA was shown to bind directly to the tyrosine kinase domain of insulin receptor, blocking the hepatocyte apoptosis induced by actinomycin D/TNF-R. Our investigation of the effects of enantiometric pure R-LA on signaling pathways and oxidative stress in cancer cell lines showed that R-LA inhibited human lung cancer cell proliferation and inhibited apoptosis of human neuroblastoma cell lines by protecting against buthionine sulfoximine (BSO)-induced glutathione depletion. ${ }^{15}$, 16

Because R-LA is found in liver and is an essential cofactor in energy metabolism, it should affect the concentrations of various metabolites in hepatocytes. Our group reported that intercellular ROS may affect insulin signaling in the H4IIEC rat hepatoma cell line. ${ }^{17}$ Although we found that treatment with R-LA for $24 \mathrm{~h}$ had a little effect on intercellular metabolite concentrations, probably due to the very rapid metabolism of R-LA (data not shown). The concentrations of some metabolites tended to be higher in DL-LA treated that in R-LA treated cells. Pharmacokinetic investigations showed that the time to reach maximum concentration $\left(\mathrm{T}_{\max }\right)$ of plasma LA concentration was around $30 \mathrm{~min}$ after oral administration of LA to humans ${ }^{18}$ and around 10 min in rats. ${ }^{19}$ Plasma LA concentrations returned to baseline $2 \mathrm{~h}$ after LA administration, indicating that LA metabolism is very rapid and that these metabolites are used or stored mainly in the liver. The aims of this study were to assess the time-course of metabolite concentrations in LA-treated rat hepatoma cells and especially to compare the effects of pure R-LA with the racemate. 


\section{Materials and Methods}

\subsection{Chemicals}

$\mathrm{R}(+)$-alpha-lipoic acid (R-LA) was purchased from Toyo Hakko Co., Ltd (Obu, Japan) and DL-alpha-lipoic acid (DL-LA) was purchased from Sigma-Aldrich Japan K.K. (Osaka, Japan). Dulbecco’s modified Eagle medium (D-MEM), L-glutamic acid, penicillin, streptomycin and phosphate buffered saline (PBS) were purchased from Wako Pure Chemical Ind., Ltd. (Osaka, Japan). Fetal bovine serum (FBS Gibco ${ }^{\circledR}$ ) was purchased from Thermo Fisher Scientific K.K. (Yokohama, Japan), methoxyamine hydrochloride was purchased from Sigma-Aldrich Japan Co. LLC. (Tokyo, Japan), and N-methyl-N-TMS-trifluoroacetamide (MSTFA) was purchased from GL Science Inc. (Tokyo, Japan). All reagents used in this study were of analytical grade or higher. Water was purified using a Milli- $Q^{\circledR}$ water purification system (Merck Millipore, Billerica, MA, USA). All chemicals were used as supplied without further purification.

\subsection{Cell culture}

H4IIEC3 cells (American Type Culture Collection, Manassas, VA, USA) were cultured in DMEM supplemented with $10 \%$ FBS, penicillin $(100 \mathrm{U} / \mathrm{mL})$ and streptomycin $(0.1 \mathrm{mg} / \mathrm{mL})$ at $37^{\circ} \mathrm{C}$ in a humidified atmosphere containing $5 \% \mathrm{CO}_{2}$. The cells ( $7^{\text {th }}$ passage cells) were cultured to $80-90 \%$ confluence in $10 \mathrm{~cm}$ dishes. After removal of the culture medium and washing with PBS, the cells were subjected to the serum starvation for $16 \mathrm{~h}$ by culturing in DMEM alone. To assess the effects of R-LA and DL-LA on hepatic metabolism, these cells were treated with the $10 \mathrm{~mL}$ DMEM medium containing $100 \mu \mathrm{M}$ R-LA or DL-LA and ethanol (maximum 0.002 vol\%) for 0.5, 1, 3, 6, 12 and $24 \mathrm{~h}$. Control samples were treated identically, but without addition of R-LA or DL-LA. The medium was removed, the cells in dishes were washed twice with PBS, and $800 \mu \mathrm{L}$ of $80 \%$ methanol was added to each dish. The cells were transferred into clean $1.5 \mathrm{~mL}$ tubes using a cell scraper and stored at $-80^{\circ} \mathrm{C}$.

\subsection{Analysis of Aqueous Metabolites}

Aqueous metabolites were analyzed using GC/MS. Metabolites were extracted and derivatized as described $^{20}$, with the following modifications. A $5 \mu \mathrm{L}$ aliquot of $0.5 \mathrm{mg} / \mathrm{mL} 2$-isopropylmalic acid (internal standard) was added to each defrosted cell sample. The samples were sonicated for $20 \mathrm{~s}$ (UR-21P, Tomy Seiko Co., Ltd., Tokyo, Japan) to break the cellular membrane, and the mixtures were incubated for 30 min at $37^{\circ} \mathrm{C}$ and centrifuged at $15,000 \times \mathrm{g}$ for $3 \mathrm{~min}$ at $4^{\circ} \mathrm{C}$. An $800 \mu \mathrm{L}$ aliquot of each supernatant was transferred to a clean 2.0-mL tube; and the residual solid sample was evaporated in a vacuum centrifuge dryer for $1 \mathrm{~h}$. To each tube containing supernatant was added $500 \mu \mathrm{L}$ of chloroform, followed by vortexing and centrifugation at $15,000 \times \mathrm{g}$ for $3 \mathrm{~min}$ at $4^{\circ} \mathrm{C}$. Each supernatant $(800 \mu \mathrm{L})$ was transferred to clear $1.5 \mathrm{~mL}$ tubes, to which was added $200 \mu \mathrm{L}$ of Milli-Q water. The tubes were vortexed and centrifuged at 15,000 $\times \mathrm{g}$ for $3 \mathrm{~min}$ at $4^{\circ} \mathrm{C}$. Each supernatant $(1000 \mu \mathrm{L})$ was transferred to a clear $1.5 \mathrm{~mL}$ tube and evaporated in a vacuum centrifuge dryer for $1 \mathrm{~h}$. These samples were subsequently freeze-dried overnight.

For oximation, $50 \mu \mathrm{L}$ of methoxyamine hydrochloride in pyridine $(20 \mathrm{mg} / \mathrm{mL})$ was added to each sample, followed by vortexing, sonication in a water bath for $20 \mathrm{~min}$, and incubation for $90 \mathrm{~min}$ at $30^{\circ} \mathrm{C}$. For trimethyl silylation, $25 \mu \mathrm{L}$ of MSTFA was added to each sample, followed by incubation at $37^{\circ} \mathrm{C}$ for 30 min and centrifugation at $15,000 \times \mathrm{g}$ for $5 \mathrm{~min}$. The resulting supernatant was transferred to an amber GC/MS vial, followed by analysis on a GCMS-QP 2010 Ultra (Shimadzu, Kyoto, Japan), using the following parameters. The column was a $30 \mathrm{~m} \times 0.25 \mathrm{~mm}$ i.d. fused silica capillary column coated with $0.25 \mu \mathrm{m}$ CP-SIL 8 CB low bleed/MS (Agilent Technologies, CA, USA). The front inlet temperature was $230^{\circ} \mathrm{C}$. The helium gas flow rate through the column was $1.12 \mathrm{~mL} / \mathrm{min}$. The column temperature was held at $80^{\circ} \mathrm{C}$ for 2 min isothermally, increased $15^{\circ} \mathrm{C} / \mathrm{min}$ to $330^{\circ} \mathrm{C}$ and held at $330^{\circ} \mathrm{C}$ for 6 min isothermally. The interface and ion source temperatures were $250^{\circ} \mathrm{C}$ and $200^{\circ} \mathrm{C}$, respectively. The MS scan started at $3.55 \mathrm{~min}$ and ended at $24.00 \mathrm{~min}$, with scans recorded over a mass range $85-500 \mathrm{~m} / \mathrm{z}$ at a scan speed of 10,000 amu/sec. Injection volume was 1 $\mu \mathrm{L}$ and split mode was 25/1 (v/v).

\subsection{GC/MS data analysis}

Data were pre-processed using MetAlign (RIKILT, Wageningen University and Research Centre, Netherland). ${ }^{21}$ AIoutput was used for peak identification, prediction, and data integration from results exported from MetAlign, user defined retention times and a library of spectra. ${ }^{22}$ AIoutput is an analysis tool for GC/MS based metabolomics written in visual basic for application (VBA, excel macro) and available in 
Microsoft Excel Windows. Peak intensity data were calculated by AIoutput based on the results of protein assays.

\subsection{Protein Assay}

Cellular proteins were assayed using the Pierce ${ }^{\circledR}$ BCA protein assay kit (Thermo Fisher Scientific K.K., Yokohama, Japan). Briefly, each residual solid sample following extraction was resuspended in $1 \mathrm{~mL}$ of 20 mM HEPES (pH 7.4), $20 \mathrm{mM} \mathrm{NaCl,} \mathrm{1 \%} \mathrm{NP40} \mathrm{and} \mathrm{1 \%} \mathrm{SDS.} \mathrm{After} 1$ min of sonication, $10 \mu \mathrm{L}$ of sample was mixed with $200 \mu \mathrm{L}$ of reaction solution (a mixture of reagents A and B prepared as described in the user guide) in 96 well plates. The sample mixtures and standard solutions (0, 250, 500, 1000 and $2000 \mu \mathrm{g} / \mathrm{mL}$ ) were incubated at $37^{\circ} \mathrm{C}$ for $30 \mathrm{~min}$, and the absorbance at $570 \mathrm{~nm}$ were measured using a Microplate Reader (Multiskan FC, Thermo Fisher Scientific K.K., Yokohama, Japan). GC/MS peak intensities were adjusted based on calculated protein concentrations.

\subsection{Statistics}

Data were expressed as means \pm SD. Dunnet's test was used to compare findings in R-LA and DL-LA treated cells with those in control cells, and Tukey's test was used to compare findings in R-LA and DL-LA treated cells. All statistical analyses were performed using Pharmaco Analyst (Three S Japan Corporation, Tokyo, Japan), with p values $<0.05$ considered statistically significant.

\section{Results}

After excluding metabolites over the upper limit of detection (e.g. phosphate), a total of 59 metabolites were detected (Table 1). Starvation of the H4IIEC3 hepatoma cell line can result in the generation of glucose through gluconeogenesis. In this study, H4IIEC3 cells were starved for $16 \mathrm{~h}$ before treatment with LA. Control cells, which were not treated with LA, should carry out gluconeogenesis and glucose consumption at the same time. (The metabolic pathway of glucose is depicted in appendix figure A1(c)).

\subsection{LA-induced hepatic central carbon profile}

\subsubsection{Glycolysis pathway}

LA has been reported to stimulate glucose uptake via both GLUT4 translocation and GLUT4 activation in hepatocytes, adipocytes and myotubes. ${ }^{7}$ Because protein-bound R-LA is a cofactor of the enzyme, pyruvate dehydrogenase, LA may affect both the beginning and end of glycolysis. We therefore evaluated the effects of R-LA and DL-LA on the cellular levels of five glycolysis metabolites (Figure 1(a) to (e)). Glucose levels did not differ significantly among the two LA-treated cells and the control cell, but tended to be lower in LA-treated than in control cells after $3 \mathrm{~h}$. The cellular level of fructose-6-phosphate was significantly lower in R-LA-treated than in DL-LA-treated and control cells at 12 and $24 \mathrm{~h}$ and tended to be lower in DL-LA-treated than in control cells. Cellular concentrations of phosphoenolpyruvate were significantly lower in LA-treated than in control cells at $12 \mathrm{~h}$, and were significantly lower in R-LA treated than in control cells at $24 \mathrm{~h}$. Because the methodology used detected pyruvate and oxaloacetic acid as the same component, they could not be distinguished. The level of pyruvate plus oxaloacetic acid was significantly lower in LA treated than in control cells at $12 \mathrm{~h}$ and tended to be lower after 3-24 h. The levels of all these central carbon metabolites changed drastically at $0.5 \mathrm{~h}$, indicating that cellular energy metabolism was very rapid and that LA affected the cellular glycolysis pathway quickly (Figure 2).

LA also affected the profile of lactic acid, which is produced from pyruvate under anaerobic conditions. Lactic acid levels were significantly lower in R-LA treated than in control cells at 6 and $24 \mathrm{~h}$ and were significantly lower in DL-LA treated than in control cells at $12 \mathrm{~h}$. Lactic acid levels tended to be lower in LA treated than in controls throughout the test period. 


\subsubsection{TCA cycle}

Protein-bound R-LA is a cofactor of the enzymes pyruvate dehydrogenase and $\alpha$-ketoacid dehydrogenase, thereby affecting the initiation and middle of the tricarboxylic acid cycle (TCA) cycle (Appendix figure A1(c)). Four metabolites of the TCA cycle were detected (Figure 1(e), (f) to (h)), the combination of citric acid and isocitric acid, fumaric acid, malic acid and the combination of pyruvate and oxaloacetic acid. Cellular citric acid and isocitric acid levels were significantly lower in cells treated with LA than in control cells at 12 and $24 \mathrm{~h}$. In control cells, citric and isocitric acid levels were significantly higher after $3 \mathrm{~h}$ than at base line $(0 \mathrm{~h})$, whereas, in LA treated cells, citric acid and isocitric acid levels did not increase. Fumaric acid levels showed a similar pattern, increasing gradually but significantly in the control group after $0 \mathrm{~h}$ and decreasing slightly at $24 \mathrm{~h}$. Fumaric acid levels in LA-treated cells were unchanged and were significantly lower than in control cells at 6,12 and $24 \mathrm{~h}$. Cellular malic acid levels showed a similar pattern, except that malic acid level at $24 \mathrm{~h}$ was significantly lower in R-LA than in DL-LA treated cells.

\section{Figure 1.}

\subsection{Enantioselective influence on amino acid profile}

\subsubsection{Asn and Asp (converted to oxaloacetic acid)}

Aspartic acid (Asp) is a metabolite in the urea cycle and participates in gluconeogenesis. It carries reducing equivalents in the malate-aspartate shuttle, involving the ready interconversion between aspartate and oxaloacetate, the oxidized (dehydrogenated) derivative of malic acid. Aspartate provides one nitrogen atom in the biosynthesis of inosine, the precursor to the purine bases. In addition to its uses in the synthesis of pyrimidine and purine, aspartic acid acts as a hydrogen acceptor in ATP synthase. LA treatment reduced Asp level at $0.5 \mathrm{~h}$, whereas control, untreated cells showed a slight increase at $0.5 \mathrm{~h}$. At every measurement point in this experiment, cellular Asp level was lower in LA-treated than in control cells (Figure 2(a)). At 12 and 24 $\mathrm{h}$, the cellular Asp level was significantly lower in R-LA treated than in DL-LA treated cells. Asparagine (Asn) was not detected in these experiments.

\subsubsection{Ala, Gly, Ser, Thr, and Cys (utilized by pyruvate)}

Amino acids such as alanine (Ala) and glycine (Gly), which are components of skeletal muscle proteins, are transported to the liver and stored or used there. A part of the amino acid threonine (Thr) is transformed to succinyl-CoA via propionyl-CoA. Enzymes mediate the transformations of threonine to glycine and to serine (Ser). Ser is biosynthesized from 3-phosphoglycerate, one of the intermediate products in the glycolysis pathway, and is transformed enzymatically into pyruvate during gluconeogenesis. The serine levels of LA-treated cells decreased at $0.5 \mathrm{~h}$, whereas those of control cells did not. Throughout treatment, serine levels remained higher in the control than in LA-treated cells. Levels of glycine and threonine, which are upstream of in gluconeogenesis, decreased at $0.5 \mathrm{~h}$ in all tested groups, but were significantly lower in R-LA-treated than in control and DL-LA-treated cells at 1, 3, 6, 12 and $24 \mathrm{~h}$. Ala concentrations decreased in all three groups at $0.5 \mathrm{~h}$ and remained lower at subsequent time points in LA-treated than in control cells. Alanine levels recovered to baseline in the control, but not in the R-LA treated, group at $24 \mathrm{~h}$ (Figure 2(b) to (e)).

\subsubsection{Pro, Arg, His, Glu, and Gln (transformed to $\alpha$-ketoglutaric acid)}

Proline (Pro), arginine (Arg), histidine (His) and glutamic acid (Glu) are transformed to glutamine (Gln) and then enzymatically converted to $\alpha$-ketoglutaric acid, which enters the TCA cycle of gluconeogenesis. 
Proline concentrations after $1 \mathrm{~h}$ were significantly lower in R-LA than in control and DL-LA-treated cells and also differed significantly in R-LA and DL-LA treated cells at 12 and $24 \mathrm{~h}$. Glutamic acid level increased at $0.5 \mathrm{~h}$ in control and DL-LA-treated cells, but remained unchanged in R-LA-treated cells, being much lower in the latter than in the other two groups. After $1 \mathrm{~h}$, glutamic acid levels in the control and DL-LA-treated cells decreased to baseline; after $3 \mathrm{~h}$, glutamic acid level increased slightly in control cells, but remained lower (near zero) in R-LA-treated cells. After $12 \mathrm{~h}$, glutamic acid level was lower in DL-LA-treated than in control cells (Figure 2(f), (g)).

\subsubsection{Val, Ile, and Met (converted to succinyl-CoA)}

Valine (Val), isoleucine (Ile) and methionine (Met) are converted to propionate-CoA, transformed to succinyl-CoA, and enter the TCA cycle; through oxaloacetic acid, they are used in gluconeogenesis. Beginning after $0.5 \mathrm{~h}$, and throughout the duration of exposure, valine and isoleucine levels were lower in cells treated with R-LA and DL-LA than in control cells (Figure 2(h), (i)).

\subsubsection{Leu, Lys, and Trp}

Leucine (Leu) and lysine (Lys) are ketogenic amino acids that can be degraded directly to acetyl-CoA. In contrast to the glucogenic amino acids, which are converted to glucose, these ketogenic amino acids are not converted to glucose. In humans, Leu and Lys are exclusively ketogenic. Tryptophan (Trp) is an essential $\alpha$-amino acid in humans. Trp is metabolized in the cytoplasm and converted to alanine, a glucogenic amino acid. In the mitochondria, Trp, like Lys, is metabolized to acetyl-CoA. Trp levels were significantly lower in R-LA-treated than in DL-LA-treated and control cells at $1 \mathrm{~h}$ and were significantly lower in DL-LA-treated than in control cells at 12 and $24 \mathrm{~h}$. The cellular Lys level and the Trp level showed the different profiles, that is because Lys is one of the ketogeneic amino acids (Figure 2(j), (k)).

\section{Figure 2.}

Table 1. Levels of metabolites in H4IIEC cells treated with R-LA, DL-LA, or untreated (control).

Values are expressed as mean of relative abundance $(\mathrm{n}=3)$. ${ }^{*} p<0.05,{ }^{* *} p<0.01$ vs. control. \#p< 0.05 , \#\#p< 0.01 vs. DL-LA. 


\subsection{PCA analysis}

Principal component analysis (PCA) is a statistical procedure, which is currently one of the most widely used methodologies to analyze metabolomic data. PCA analysis of metabolomic data using SIMCA (Umetrics, Malmö, Sweden) software showed that the cells treated with DL-LA and R-LA could be clearly segregated from the non-treated control cells at 0.5, 6 and $12 \mathrm{~h}$ (Figure 3). At these three time points, the performance parameters of R2X[1] were $0.469,0.681$, and 0.738 , respectively, and the parameters at R2X[2] were 0.323 , 0.185 , and 0.190 , respectively, indicating that the model was reliable and highly predictive. This analysis identified 32 metabolites at $6 \mathrm{~h}$ and 35 at $12 \mathrm{~h}$ with $\mathrm{R} 2>0.8$. Their Q2 values were also high, further indicating that the PCA model could well explain the data set and have good predictive ability.

\section{Figure 3.}

\section{Discussion}

Intracellular glucose is phosphorylated to glucose 6-phosphate; isomerization of glucose 6-phosphate yields fructose 6-phosphate (F6P), which lies within the glycolysis metabolic pathway. In untreated (control) H4IIEC3 cells, F6P levels were higher at $0.5 \mathrm{~h}$ than at baseline, decreasing at $6 \mathrm{~h}$, and increasing at 12 and 24 h. R-LA treatment kept the cellular F6P level significantly lower than in control cells at 12 and $24 \mathrm{~h}$. Phosphoenolpyruvate (PEP) is another intermediate metabolite in the glycolysis metabolic pathway. Metabolism of PEP to pyruvate by pyruvate kinase generates one molecule of adenosine triphosphate (ATP) via substrate-level phosphorylation. In contrast to most normal cells, in which energy is produced by a comparatively low rate of glycolysis followed by oxidation of pyruvate in mitochondria, energy in most cancer cells results from the Warburg effect, in which energy is produced predominantly by a high rate of glycolysis followed by lactic acid fermentation in the cytosol. ${ }^{23-26}$ The metabolic profiles of F6P, PEP and lactic acid in these H4IIEC3 cells are consistent with the Warburg effect. Although the profile of pyruvate should be similar to that of lactic acid, the methodology we used could not distinguish between pyruvate and oxaloacetic acid. LA has been reported to reduce cell viability/proliferation, glucose uptake and lactate production, and to increase apoptosis, in tumor cell lines. ${ }^{9}$ We found that R-LA treatment of H4IIEC3 cells reduced glucose uptake, ultimately reducing the production of lactic acid. These results indicate that R-LA inhibited the glycolysis metabolic pathway in these H4IIEC3 cells.

The intermediate metabolites of the TCA cycle, including pyruvate and oxaloacetic acid, citric acid and isocitric acid, fumaric acid, and malic acid, showed lower relative abundance than the metabolites of the glycolysis pathway, such as lactic acid, in H4IIEC3 cells. These results indicate that a greater amount of pyruvate was converted to lactic acid than taken up by the mitochondria. This was due to the Warburg effect, which resulted in a change from the oxidative (pyruvate/acetylCoA) to the non-oxidative (pyruvate/lactate) metabolic pathway. Next, we focused our attention on the level of pantothenate. Pantothenate is well known to be involved in the synthesis of coenzyme A (CoA) and CoA is also the cofactor of pyruvate dehydrogenase and $\alpha$-ketoglutaric acid in the TCA cycle. The results of pantohenate are also consistent with this observation. R-LA treated cellular pantothenate level is significantly lower than those of control and DL-LA treated cell, which means the predominance of the non-oxidative pathway (pyruvate/lactate) by R-LA treatment (Figure 2(l)).

Assessment of amino acid metabolism showed that LA treatment reduced the amounts of amino acids in these cells, beginning at $0.5 \mathrm{~h}$. Serine and alanine, which are biosynthesized in the body and converted to pyruvate, were significantly decreased by LA treatment. Glycerate 3-phosphate (GP), one of the intermediate metabolites in the glycolysis pathway, is a precursor of serine. Because LA treatment inhibited the glycolysis pathway, the GP level was low, reducing the serine level in these cells. Gly and Thr lie upstream of serine in the Thr-Gly-Ser pathway. Cellular concentrations of Gly, Thr and Ser were significantly lower in R-LA treated than in untreated (control) and DL-LA treated cells, indicating enantio-selectivity for these three amino acids. The conversions of Thr to Gly and of Gly to Ser are catalyzed by serine hydroxymethyltransferase (SHMT). These results indicated that R-LA, but not DL-LA, down-regulated the 
Thr-Gly-Ser pathway, inhibiting related enzymes such as SHMT. Taken together, these results indicated that R-LA treatment inhibited glycolysis, lactic acid production and the Thr-Gly-Ser pathway, ultimately inhibiting gluconeogenesis, in H4IIEC3 cells.

In both untreated and DL-LA treated cells, Glu concentrations were markedly higher at $0.5 \mathrm{~h}$ than at baseline, with both decreasing at $1 \mathrm{~h}$. These findings may be due to exchange of the culture medium, which contained a high concentration of glutamic acid. Just after the exchange of medium, H4IIEC3 cells absorbed Glu rapidly. R-LA treatment, however, resulted in low Glu concentrations throughout the experiment. This result can be explained by considering the level of pyroglutamic acid, which is derived from glutathione by the action of an enzyme, $\gamma$-glutamyl cyclotransferase. Pyroglutamic acid is the precursor of glutamic acid. R-LA treatment kept the cellular pyroglutamic acid level significantly lower than those of control and DL-LA treated cell at 6-24 h (Figure 2(m)).

Many metabolites in H4IIEC3 cells showed rapid responses to LA treatment. Measurements of cellular metabolite levels 0.5 and $1 \mathrm{~h}$ after LA treatment were important in determining initial responses, whereas measurements at 6-24 h were important in determining the overall effect of LA treatment. We therefore classified the detected metabolites into the four major and four minor types (Appendix, Table A1). Most of the metabolites classified into the Control > DL-LA = R-LA type were TCA cycle components and saccharides. Most of the metabolites classified into the Control = DL-LA > R-LA type were amino acids. The metabolites classified into the Control > DL-LA > R-LA type included amino acids and glycolysis metabolites. We also identified 42 metabolites with quick responses to LA treatment (Appendix, Table A2), as shown by statistically significant difference between LA treated and untreated cells at 0.5 and/or $1 \mathrm{~h}$. These results indicate that LA rapidly affects components of the glycolysis and amino acid pathways. Furthermore, R-LA and DL-LA did not have the same effects on metabolic pathways in H4IIEC3 cells.

\section{Conclusions}

PCA analysis was able to differentiate among the three groups of H4IIEC3 cells, those treated with R-LA and DL-LA and untreated cells, indicating that these experiments were well designed in assessing enantioselectivity and effect. The profiles of F6P, PEP and lactic acid in these cell types were very similar and indicated that the Warburg effect was observed under these experimental conditions. The time-course profiles of the cellular level of metabolites in the glycolysis pathway suggested that R-LA treatment decreased glucose uptake and the production of lactic acid, thus inhibiting the glycolysis metabolic pathway in H4IIEC3 cells. Pyruvate uptake into mitochondria would be lower than pyruvate conversion to lactic acid, because the Warburg effect results in a shift of oxidative (pyruvate/acetyl-CoA) to non-oxidative (pyruvate/lactate) metabolism. LA treatment reduced the cellular metabolites of the TCA cycle, but enantioselectivity was not observed. R-LA, but not DL-LA, reduced cellular Gly and Thr levels. Ser levels were lower in LA treated than in control cells, and were lower in R-LA treated than in DL-LA treated cells. Taken together, these results indicated that R-LA could down-regulate the Thr-Gly-Ser pathway but DL-LA could not (Figure 4). This study therefore indicated that R-LA treatment inhibited the glycolysis pathway, lactic acid production and the Thr-Gly-Ser pathway, resulting in the inhibition of gluconeogenesis in starved H4IIEC3 cells. Moreover, these findings may explain the mechanism by which LA induces apoptosis in hepatoma cells. ${ }^{12}$

\section{Figure 4.}

Acknowledgments: We would like to thank Changshu Fushilai Medicine \& Chemical Co., Ltd and Toyo Hakko for their donation of R(+)-alpha-lipoic acid. This work (S. M.) was partially supported by a Grant-in-Aid for Exploratory Research from Japan Society for the Promotion of Science.

Author disclosure statement: N.I., K.T., G.R. and S.M. designed the study and contributed to preparation of the manuscript; N.I., K.C., Y.A., Y.Y. and H.Y. prepared cells and conducted GC/MS analysis; N.I., K.C., Y.A., and S.M. performed the PCA analysis and calculated the data. All of the authors have read and approved the final manuscript. 


\section{Abbreviations}

The following abbreviations are used in this manuscript:

G6P, glucose-6-phosphate; F6P, flucrose-6-phosphate; PEP, phophoenolpyruvic acid; AcCoA, acetyl-CoA; Cit, citrate; IsoCit, isocitrate; Akg, $\alpha$-ketoglutarate; Suc, succinyl-CoA; Fum, fumarate; Mal, malate; Oxa, oxaloacetic acid; Lac, lactate; Gln, glutamine; Glu, glutamic acid; PDH, pyruvate dehydrogenase; ADH, $\alpha$-ketoglutarate dehydrogenase; SDH, succinate dehydrogenase; LDH, lactate dehydrogenase; GDH, glutamate dehydrogenase; GLN, glutaminase; ALT, alanine transaminase; SDR, serine dehydratase; SHMT, serine hydroxymethyltransferase; GP, glycerate 3-phosphate.

\section{Appendix}

Figure A1. The chemical structures of (a) R-alpha-lipoic acid and (b) S-alpha-lipoic acid and (c) metabolic pathway of glucose. The asterisk indicates the chiral center. Abbreviations: G6P, glucose-6-phosphate; F6P, fructose-6-phosphate; PEP, phophoenolpyruvate; AcCoA, acetyl-CoA; Cit, citrate; IsoCit, isocitrate; Akg, $\alpha$-ketoglutarate; Suc, succinyl-CoA; Fum, fumarate; Mal, malate; Oxa, oxaloacetic acid; Lac, lactate; Gln, glutamine; Glu, glutamic acid; PDH, pyruvate dehydrogenase; $\mathrm{ADH}, \alpha$-ketoglutarate dehydrogenase; SDH, succinate dehydrogenase; $\mathrm{LDH}$, lactate dehydrogenase; GDH, glutamate dehydrogenase; GLN, glutaminase; ALT, alanine transaminase; SDR, serine dehydratase.

Table A1. Types of time-course changes in metabolite levels in LA treated H4IIEC cells. Inequalities were defined as significant differences in at least two of the latter 3 time points at 6, 12 and $24 \mathrm{~h}$. Equalities were defined as absence of significant differences among the three groups at these time points.

Table A2. Metabolites responding rapidly to LA treatment.

\section{References}

1. $\quad$ Reed LJ: From lipoic acid to multi-enzyme complexes. Protein Sci 1998;7: 220-224.

2. Nikolai S, Huebbe P, Metges CC, et al: R-a lipoic acid g-cyclodextrin complex increases energy expenditure: A 4-month feeding study in mice. Nutrition 2014;30: 228-233.

3. Nikolai S, Pallauf K, Huebbe P, Rimbach G: Energy restriction and potential energy restriction mimetics. Nutr Res Rev 2015;26:100-120.

4. Koriyama Y, Nakayama Y, Matsugo S, Kato S: Protective effect of lipoic acid against oxidative stress is mediated by Keap1/Nrf2-dependent heme oxygenase-1 induction in the RGC-5 cell line. Brain Res 2013;1499:145-157.

5. Koriyama Y, Nakayama Y, Matsugo S, et al: Anti-inflammatory effects of lipoic acid through inhibition of GSK-3 beta in lipopolysaccharide-induced BV-2 microglial cells. Neurosci Res 2013;77:87-96.

6. Packer L, Kraemer K, Rimbach G: Molecular aspects of lipoic acid in the prevention of diabetes complications. Nutrition 2001;17:888-895.

7. Konrad D, Somwar R, Sweeney G, et al: The antihyperglycemic drug alpha-lipoic acid stimulates glucose uptake via both GLUT4 translocation and GLUT4 activation: potential role of p38 mitogen-activated protein kinase in GLUT4 activation. Diabetes 2001;50:1464-71.

8. Estrada DE, Ewart HS, Tsakiridis T, et al: Stimulation of glucose uptake by the natural coenzyme $\alpha$-lipoic acid/thioctic acid: Participation of elements of the insulin signaling pathway. Diabetes 1996;45:1798-1804.

9. Feuerecker B, Pirsig S, Seidl C, et al: Lipoic acid inhibits cell proliferation of tumor cells in vitro and in vivo. Cancer Biol Ther 2012;13:1425-1435.

10. Wenzel U, Nickel A, Daniel H: Alpha-lipoic acid induces apoptosis in human colon cancer cells by increasing mitochondrial respiration with a concomitant O2-*-generation. Apoptosis 2005;10:359-68.

11. Choi SY, Yu JH, Kim H: Mechanism of alpha-lipoic acid-induced apoptosis of lung cancer cells. Ann N Y Acad Sci 2009;1171:149-55. 
154

155

156

157

158

159

160

161

162

163

164

165

166

167

168

169

170

171

172

173

174

175

176

177

178

179

180

181

182

183

184

185

186

187

188

189

190

191
12. Shi D, Liu H, Stern JS, Yu P, Liu S: Alpha-lipoic acid induces apoptosis in hepatoma cells via the PTEN/Akt pathway. FEBS Letters 2008;582:1667-1671.

13. Walgren JL, Amani Z, McMillan JM, Locher M, Buseet MG: Effect of R- $\alpha$-Lipoic acid on pyruvate metabolism and fatty acid oxidation in rat hepatocytes. Metabolism 2004;53:165-173.

14. Diesel B, Kulhanek-Heinze S, Ho“ltje M, et al: Alpha-lipoic acid as a directly binding activator of the insulin receptor: Protection from hepatocyte apoptosis. Biochemistry 2007;46:2146-2155.

15. Yamada T, Hashida K, Takarada-Iemata M, Matsugo S, Hori O: $\alpha$-Lipoic acid (LA) enantiomers protect SH-SY5Y cells against glutathione depletion. Neurochem Int 2011;59:1003-1009.

16. Michikoshi H, Nakamura T, Sakai K, et al: $\alpha$-Lipoic acid-induced inhibition of proliferation and met phosphorylation in human non-small cell lung cancer cells. Cancer Lett 2013;13: S0304-3835.

17. Iwakami S, Misu H, Takeda T, et al: Concentration-dependent dual effect of hydrogen peroxide on insulin signal transduction in H4IIEC hepatocytes. PLOS ONE 2011;6:e27401.

18. Hermann, R, Mungo J, Cnota PJ, Ziegler D: Enantiomer-selective pharmacokinetics, oral bioavailability, and sex effects of various alpha-lipoic acid dosage forms. Clin Pharmacol 2014;6:195-204.

19. Uchida R, Iwamoto K, Nagayama S, et al: Effect of $\gamma$-cyclodextrin inclusion complex on the absorption of R- $\alpha$-lipoic acid in rats. Int J Mo l Sci 2015;16:10105-10120.

20. Tsugawa H, Bamba T, Shinohara M, et al: Practical non-targeted gas chromatography mass spectrometry-based metabolomics platform for metabolic phenotype analysis. J Biosci Bioeng 2011;112:292-298.

21. Lommen A: MetAlign: Interface-driven, versatile metabolomics tool for hyphenated full-scan mass spectrometry data preprocessing. Anal Chem 2009;81:3079-3086.

22. Tsugawa H, Tsujimoto Y, Arita M, Bamba T, Fukusaki E: GC/MS based metabolomics: development of a data mining system for metabolite identification by using soft independent modeling of class analogy (SIMCA). BMC Bioinformatics 2011;12:131.

23. Alfarouk KO, Verduzco D, Rauch C, et al: Glycolysis, tumor metabolism, cancer growth and dissemination. A new pH-based etiopathogenic perspective and therapeutic approach to an old cancer question. Oncoscience 2014;1: 777-802.

24. Alfarouk KO, Muddathir AK, Shayoub ME: Tumor acidity as evolutionary spite. Cancers 2011;3:408-414.

25. Gatenby RA, Gillies RJ: Why do cancers have high aerobic glycolysis?. Nature Reviews Cancer 2004;4:891-899.

26. Kim JW, Dang CV: Cancer's molecular sweet tooth and the Warburg effect. Cancer Res 2006;66:8927-8930.

\section{Correspondence:}

Seiichi Matsugo, Prof., Ph.D.

e-mail: s-matsugoh@se.kanazawa-u.ac.jp

Tel.: +81-76-264-6219 ; Fax: +81-76-234-4829. 
Figure Legend

Figure 1. Time-course of the relative abundance of the glycolysis metabolites (a) glucose, (b) fructose-6-phosphate, (c) phosphoenolpyruvate, (d) lactic acid and (e) pyruvate and oxaloacetic acid, and the TCA cycle metabolites (e), (f) citric acid and isocitric acid, (g) fumaric acid and (h) malic acid in H4IIEC3 cells following treatment with R-LA, DL-LA, or no treatment (control). Values are expressed as mean \pm SD $(n=3)$. ${ }^{*} \mathrm{p}<0.05,{ }^{* *} \mathrm{p}<0.01$ vs. control. \#p $<0.05, \# \# \mathrm{p}<0.01$ vs DL-LA.

Figure 2. Time-course of the relative abundance of the amino acids (a) aspartic acid, (b) serine, (c) glycine, (d) threonine, (e) alanine, (f) proline, (g) glutamic acid, (h) valine, (i) isoleucine acid, (j) lysine, (k) tryptophan, (l) pantothenate and (m) pyroglutamic acid in H4IIEC3 cells following treatment with R-LA, DL-LA, or no treatment (control). Values are expressed as mean $\pm \mathrm{SD}(\mathrm{n}=3) .{ }^{*} \mathrm{p}<0.05$, ${ }^{* *} \mathrm{p}<0.01$ vs. control. $\# \mathrm{p}<0.05$, $\# \# \mathrm{p}<0.01$ vs DL-LA.

Figure 3. PCA scoring plots of the control (black squares), DL-LA treated (blue circles) and R-LA treated (red rhombuses) cells at (a) $0.5 \mathrm{~h}$, (b) $6 \mathrm{~h}$, (c) and $12 \mathrm{~h}$. The x- and y-axes indicate the first principal and second principal components, respectively.

Figure 4. Proposed metabolic pathways by which R-LA inhibits gluconeogenesis. (a) Inhibition of glycolysis and lactic acid production; (b) Inhibition of the Thr-Gly-Ser pathway. Abbreviations: Lac, lactate; Thr, threonine; Gly, glycine; Ser, serine; Ala, alanine; LDH, lactate dehydrogenase; ALT, alanine transaminase; SDR, serine dehydratase; GP, glycerate 3-phosphate; SHMT; serine hydroxymethyltransferase. 
(a)

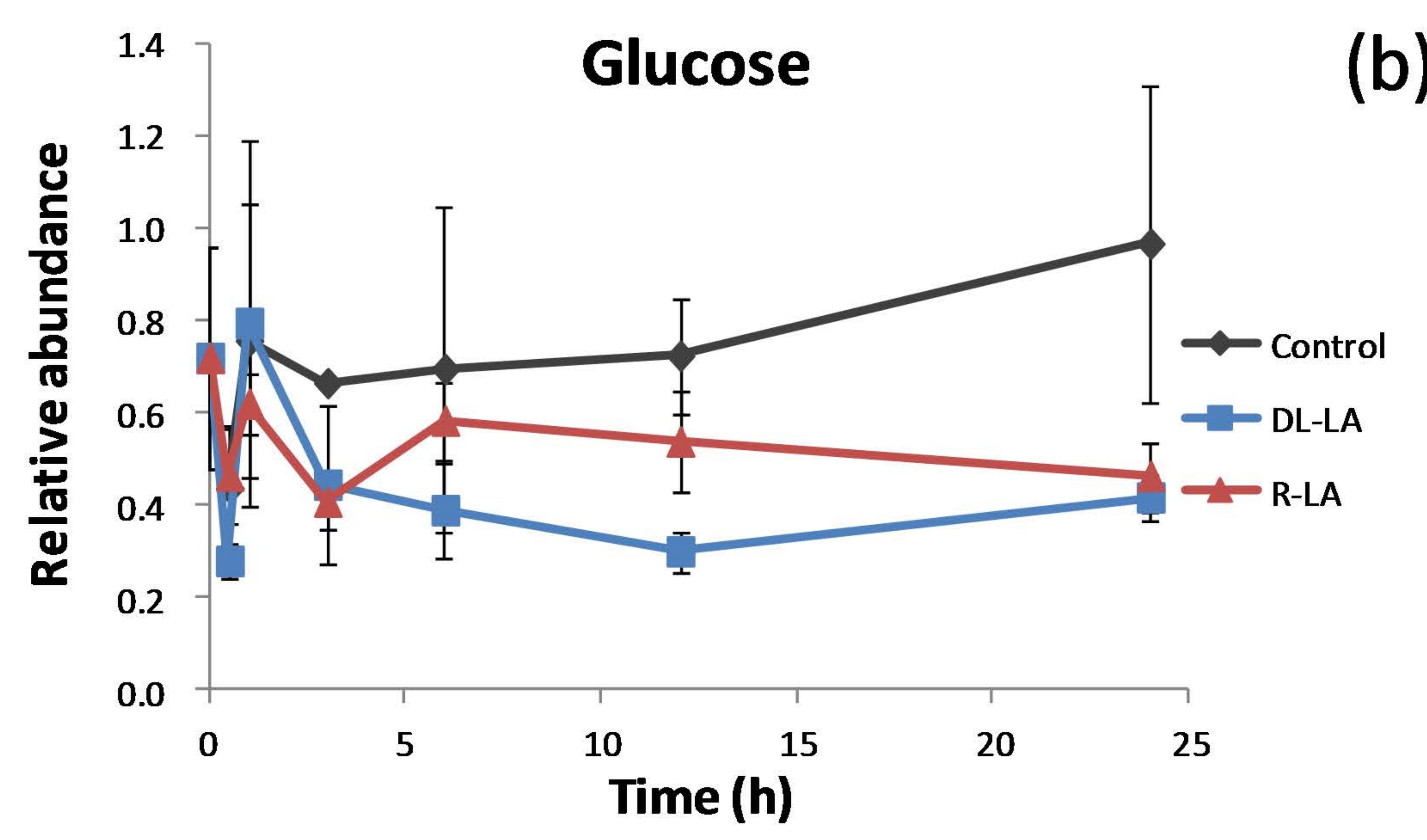

(c)

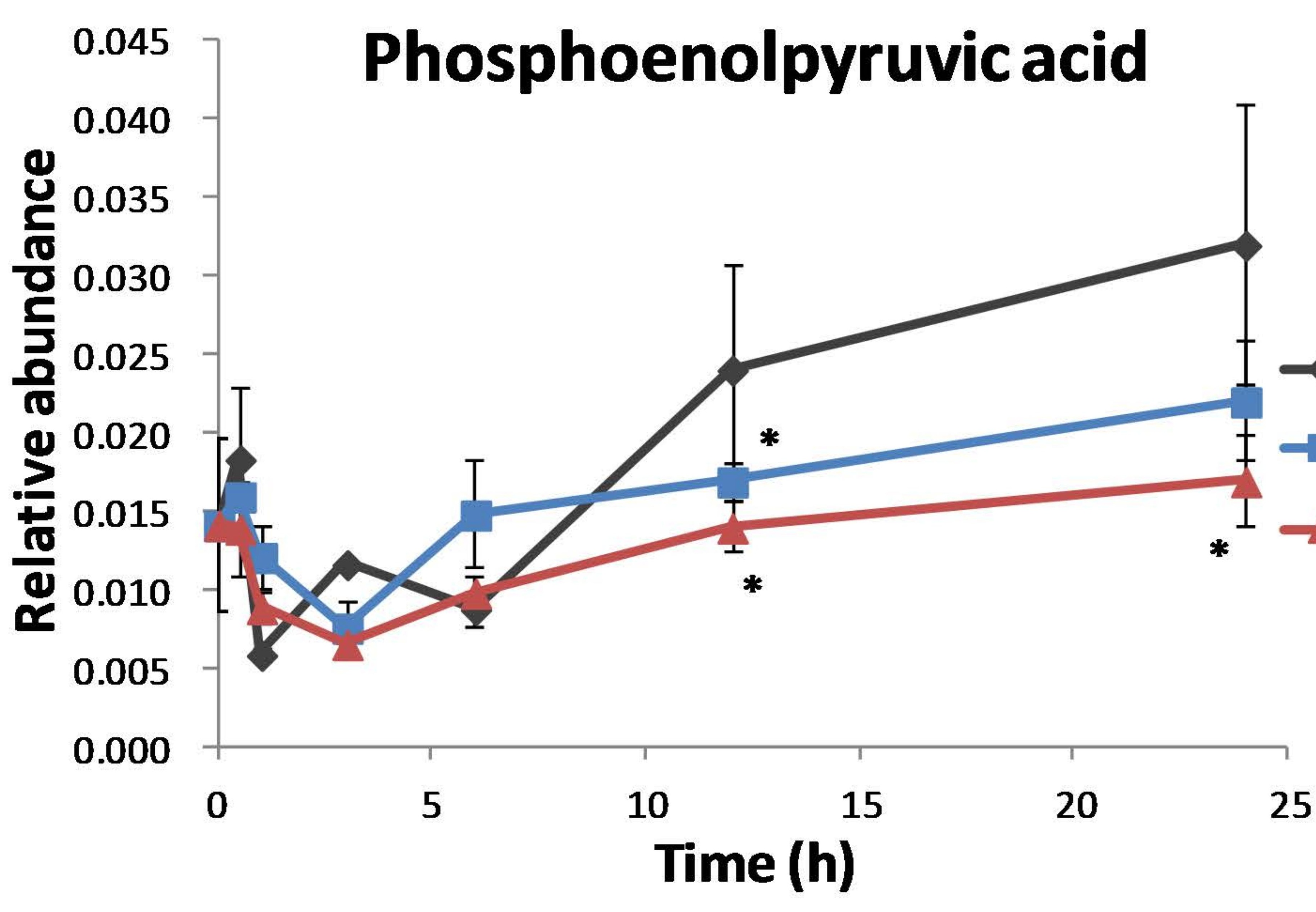

(e)

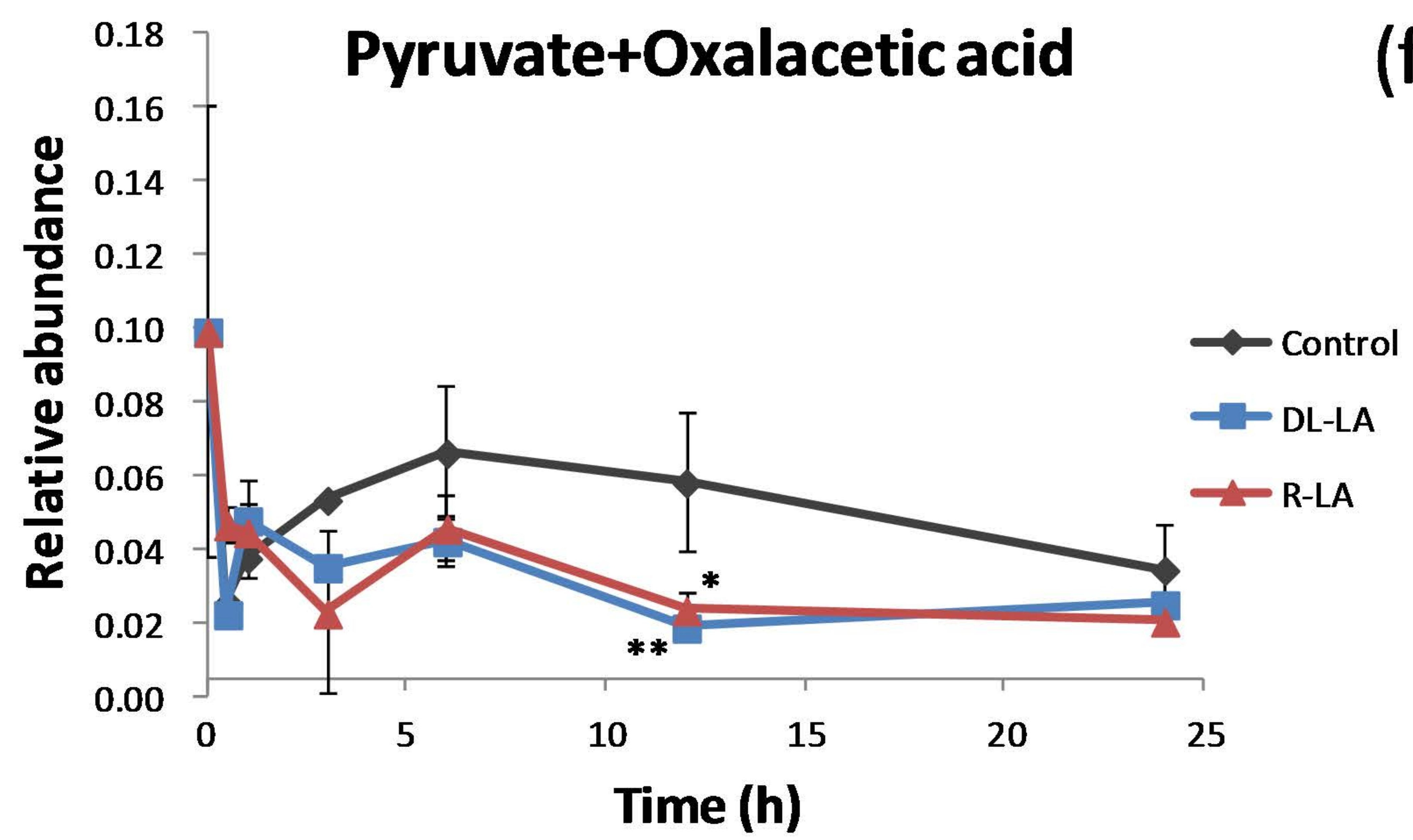

(g)

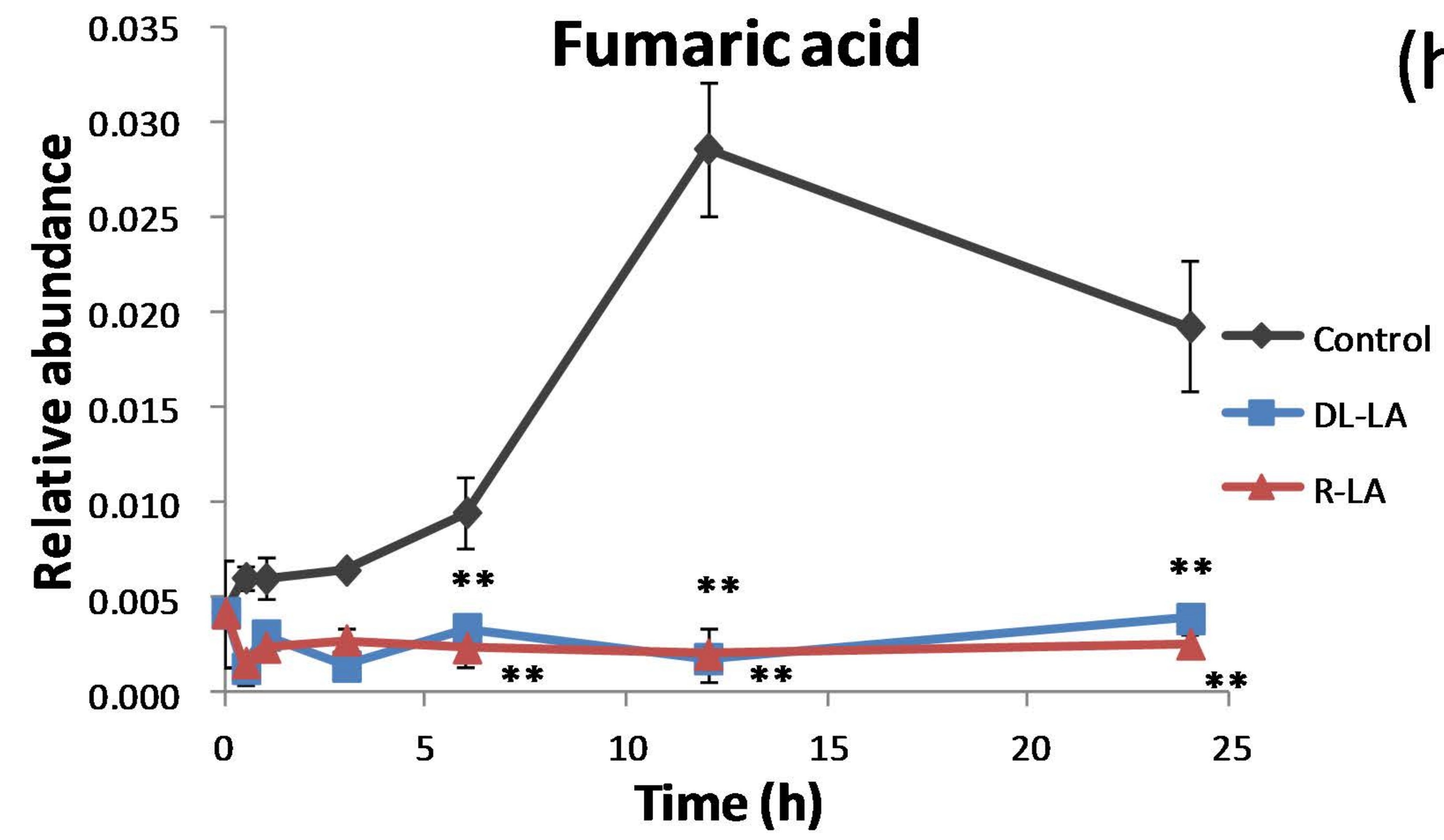

(b)

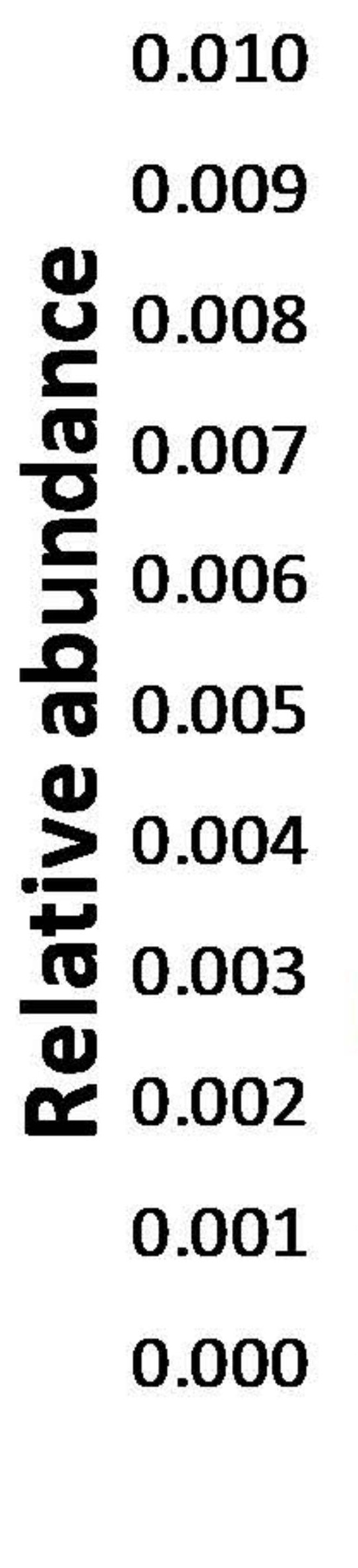

(d)

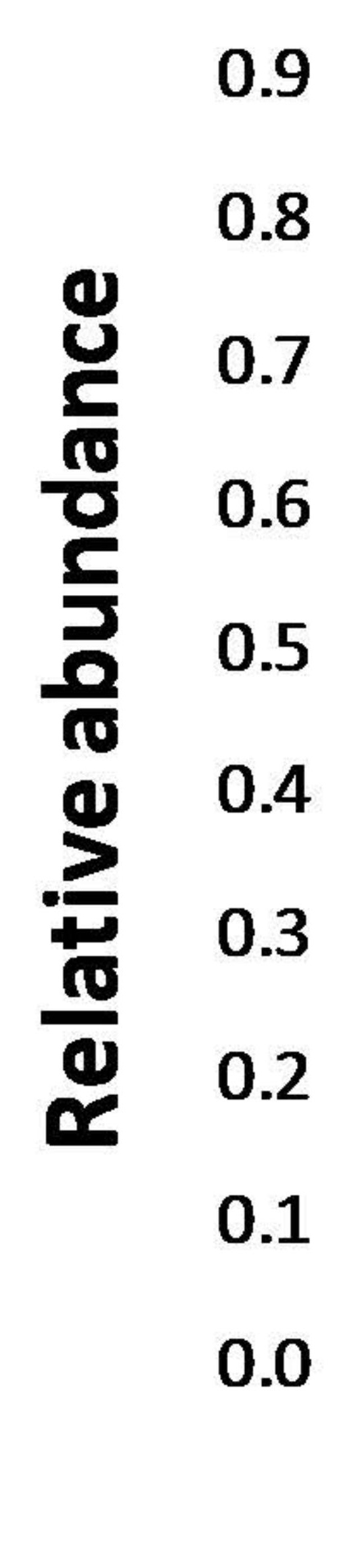

(f)

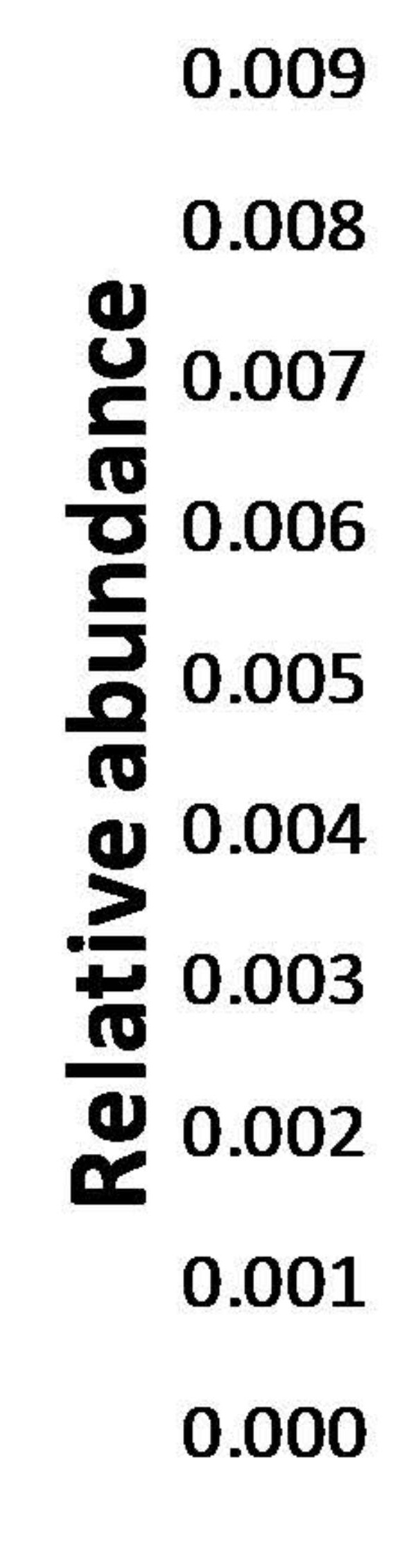

(h)

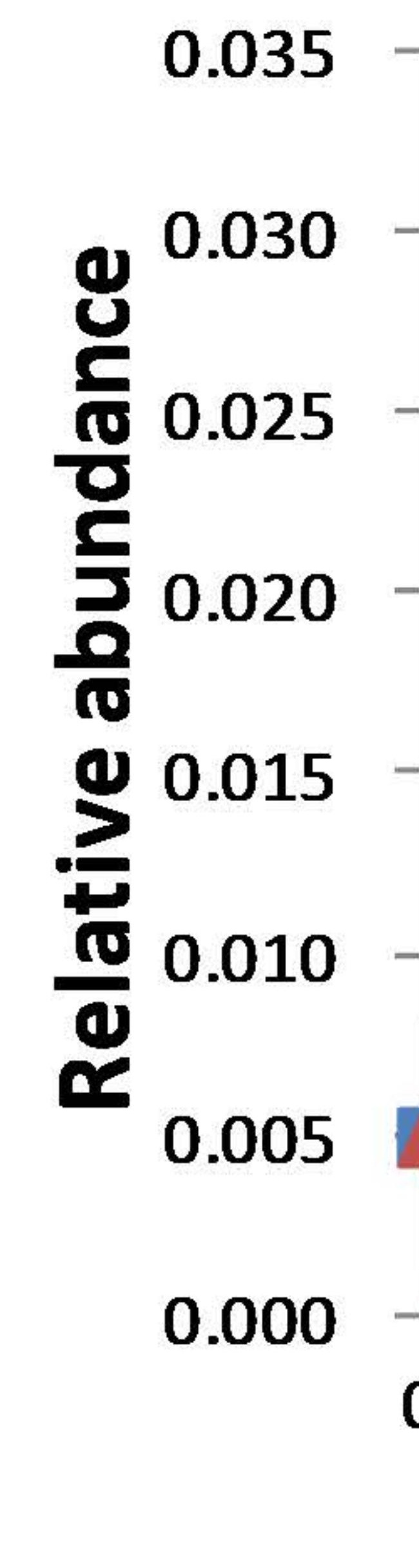

Fructose-6-Phosphate
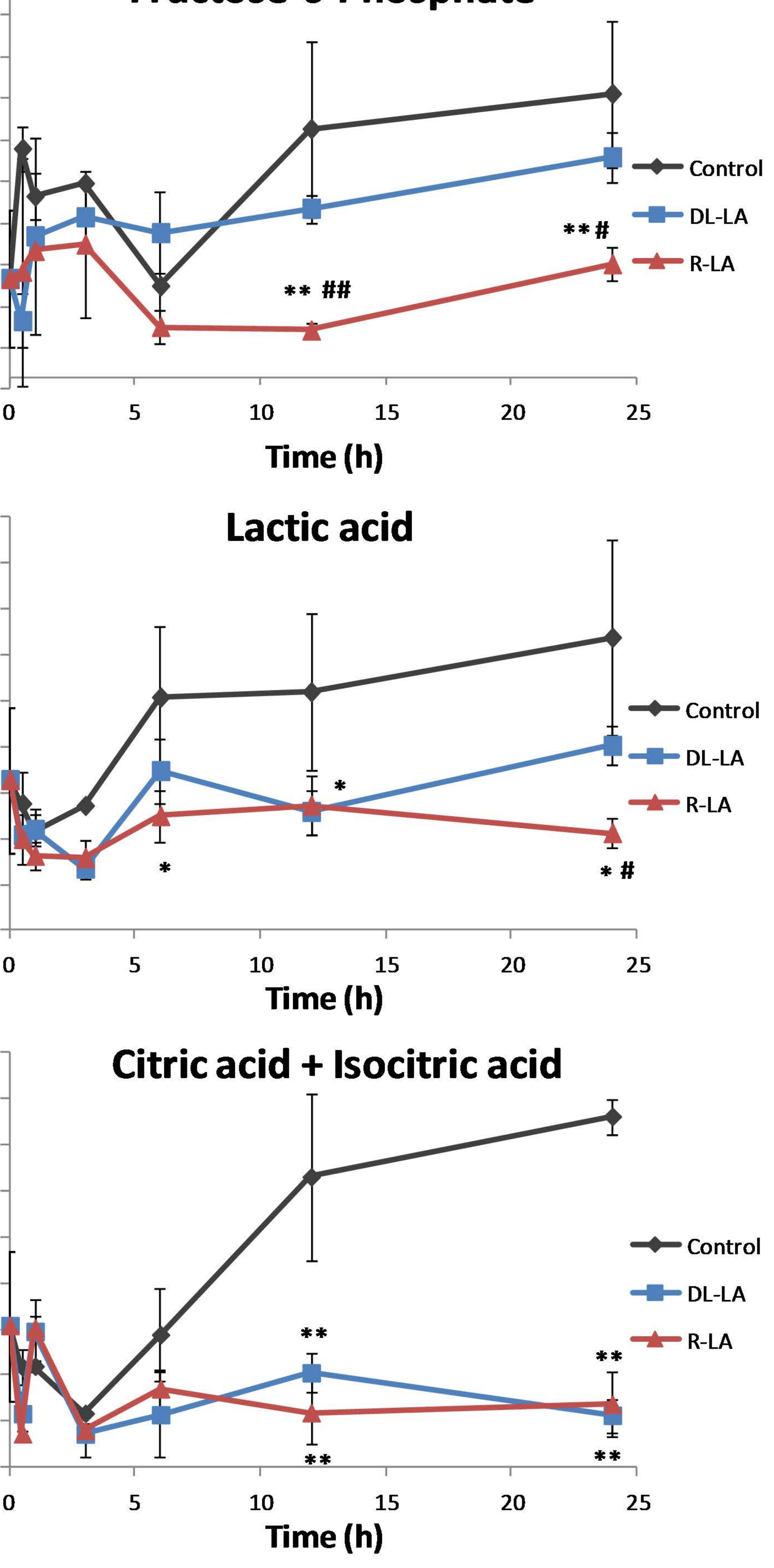

Malic acid

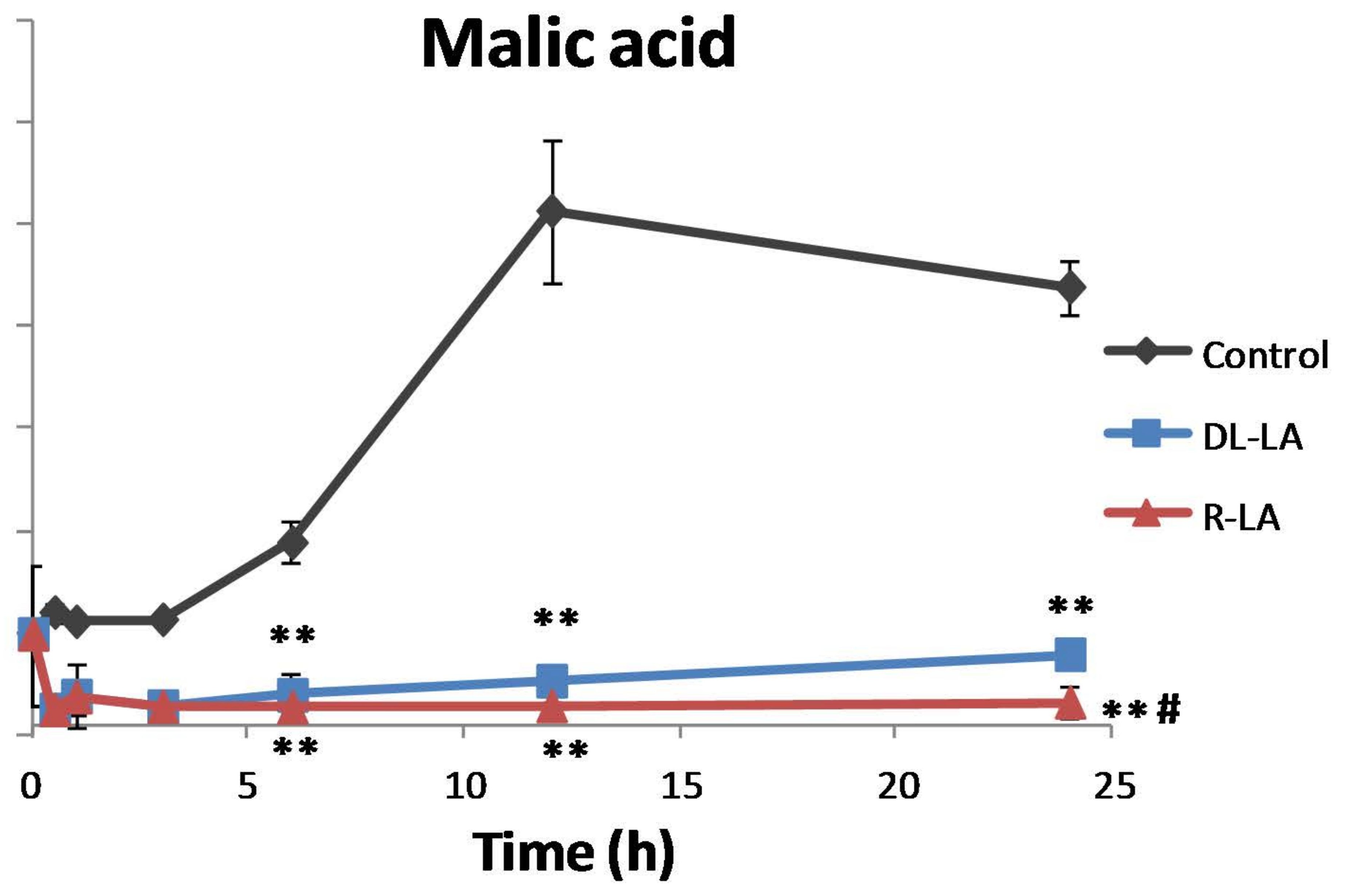


(a) 0.25

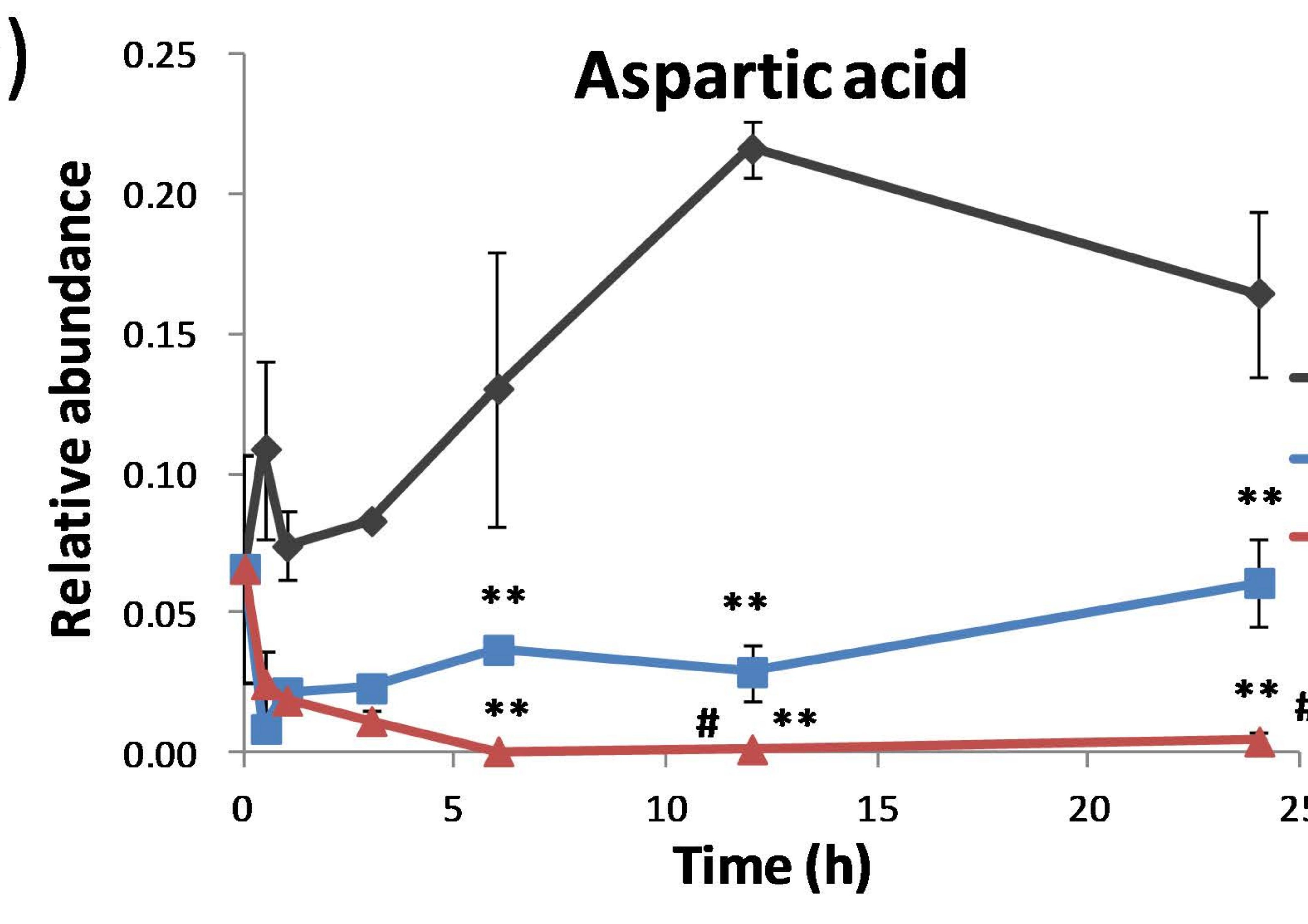

(d)

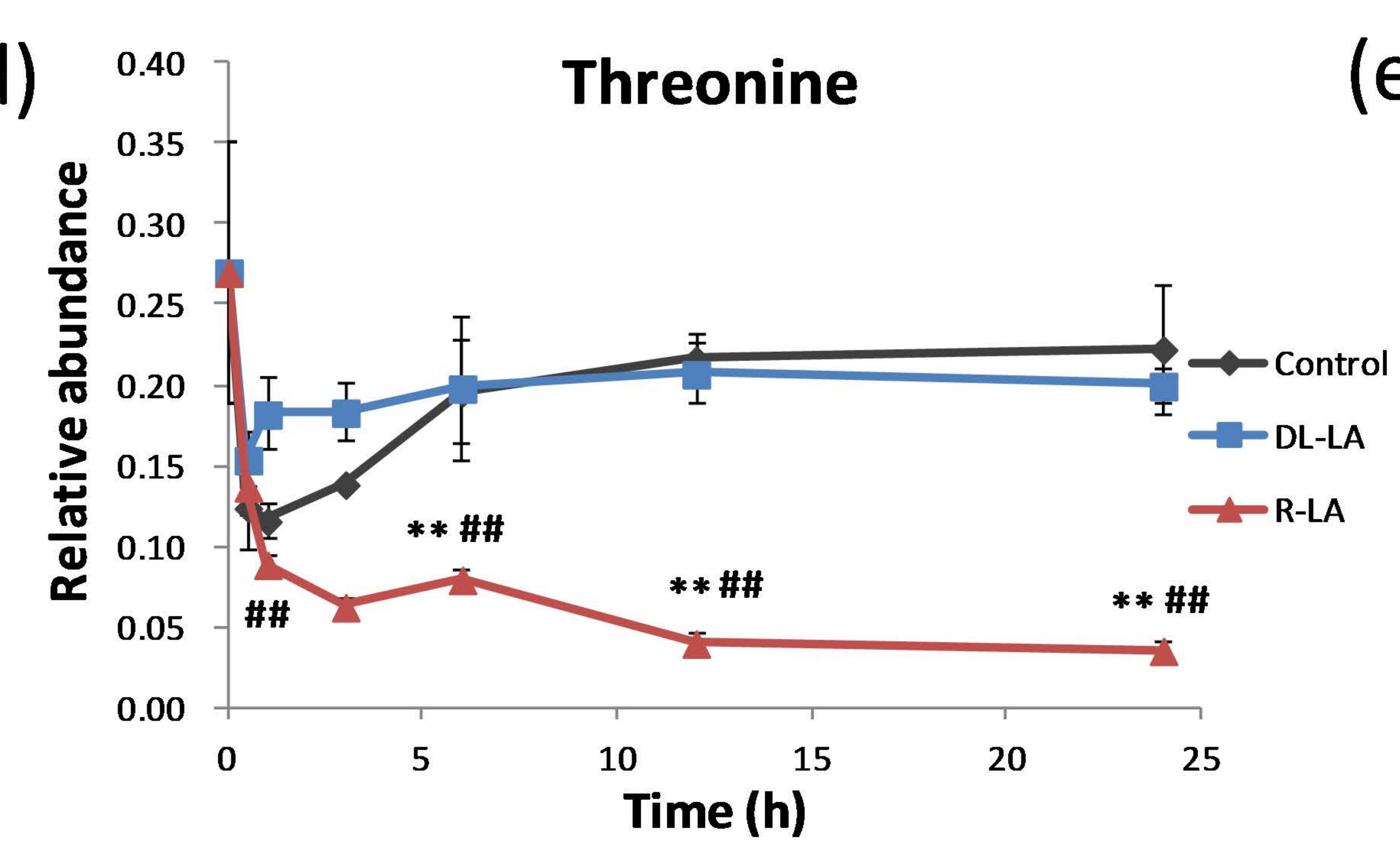

(g)

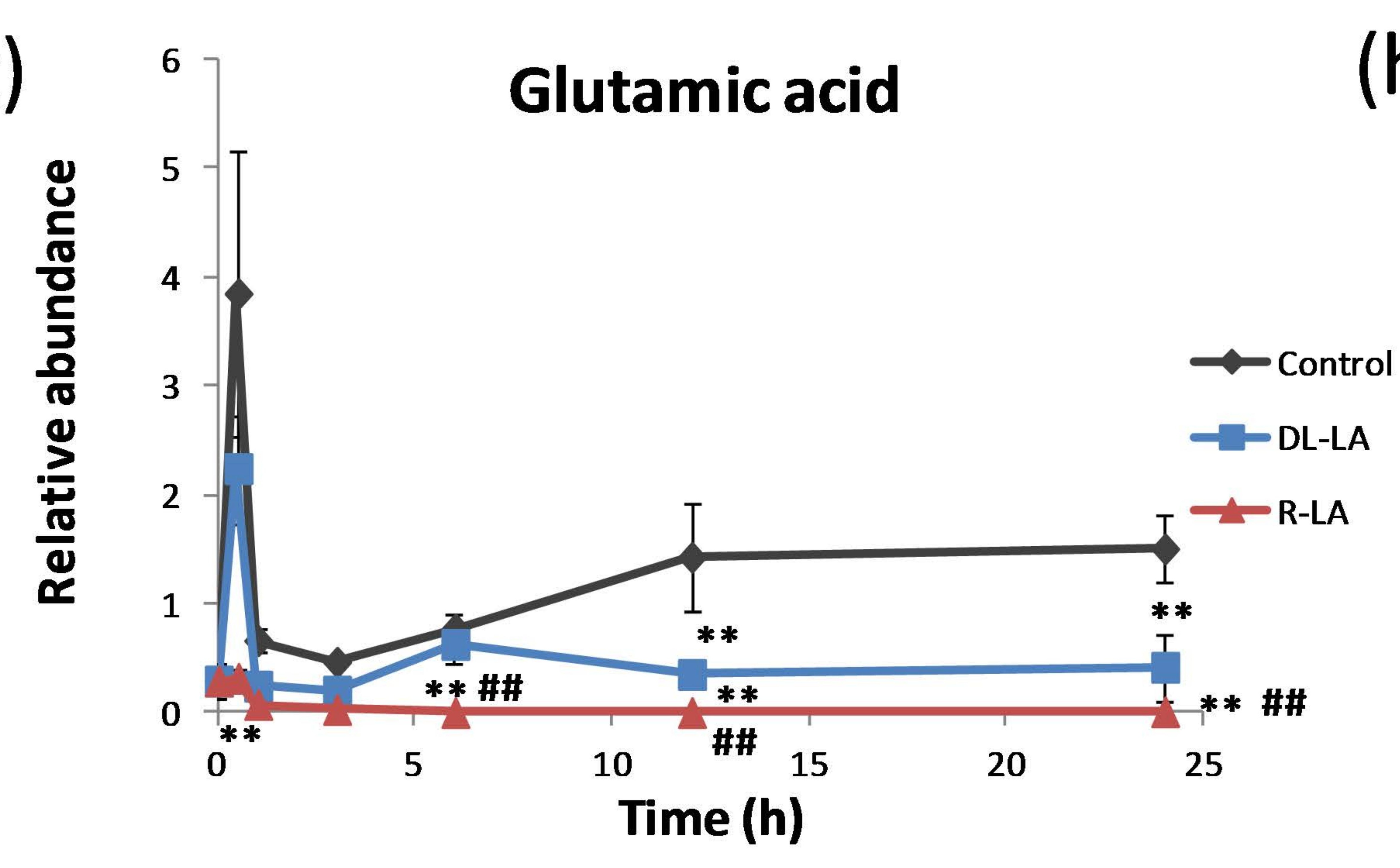

(j)

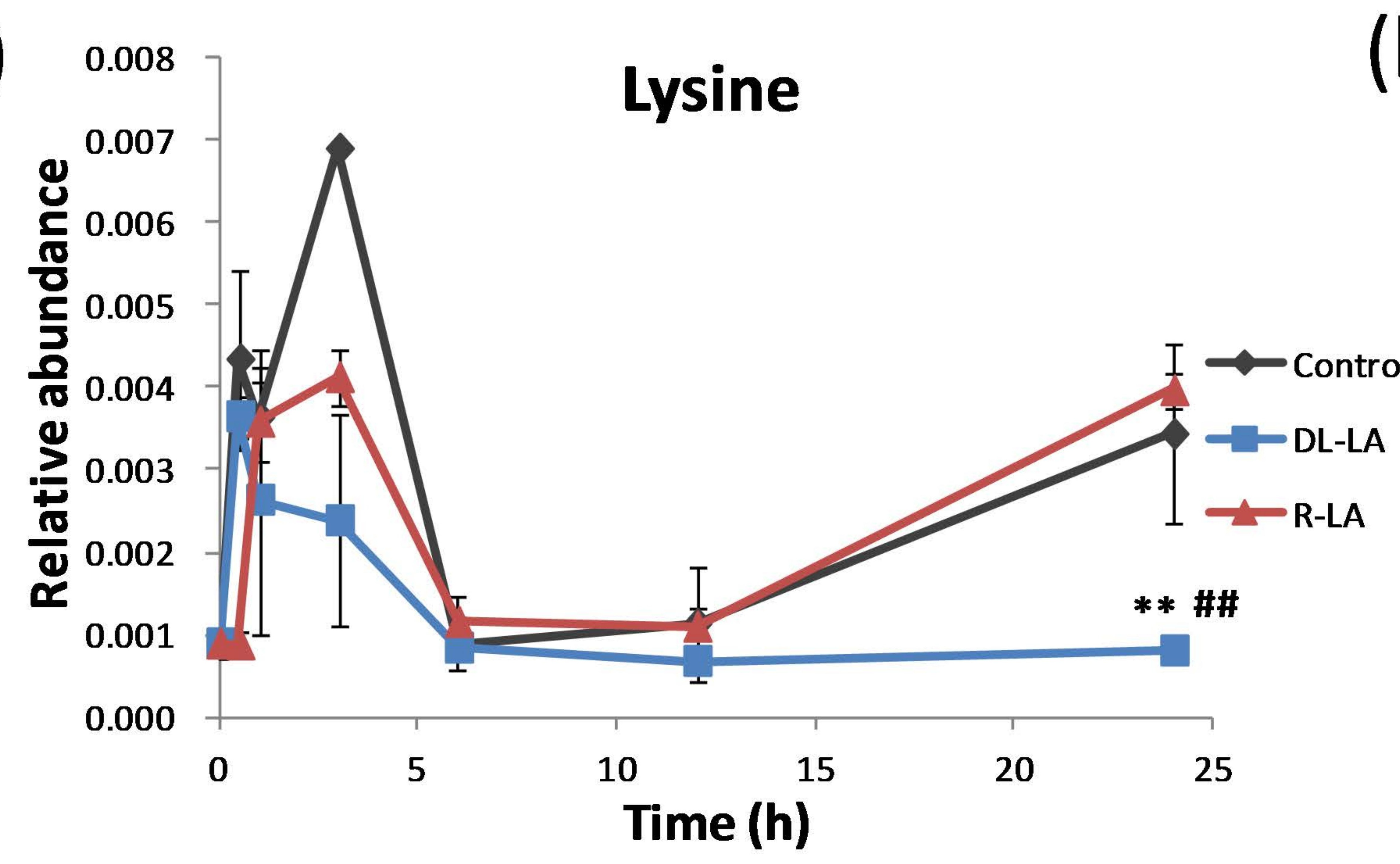

(I)

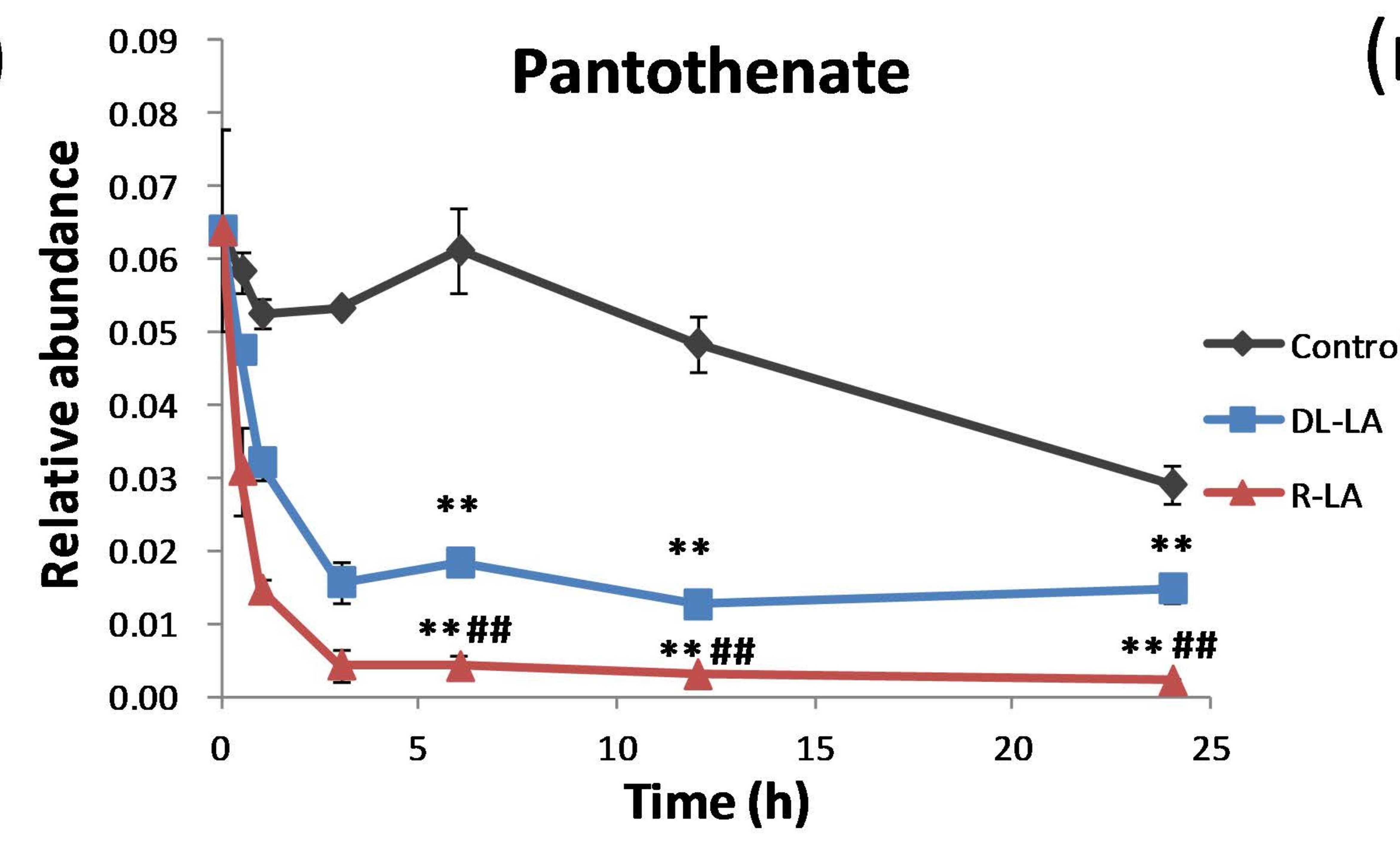

(b)

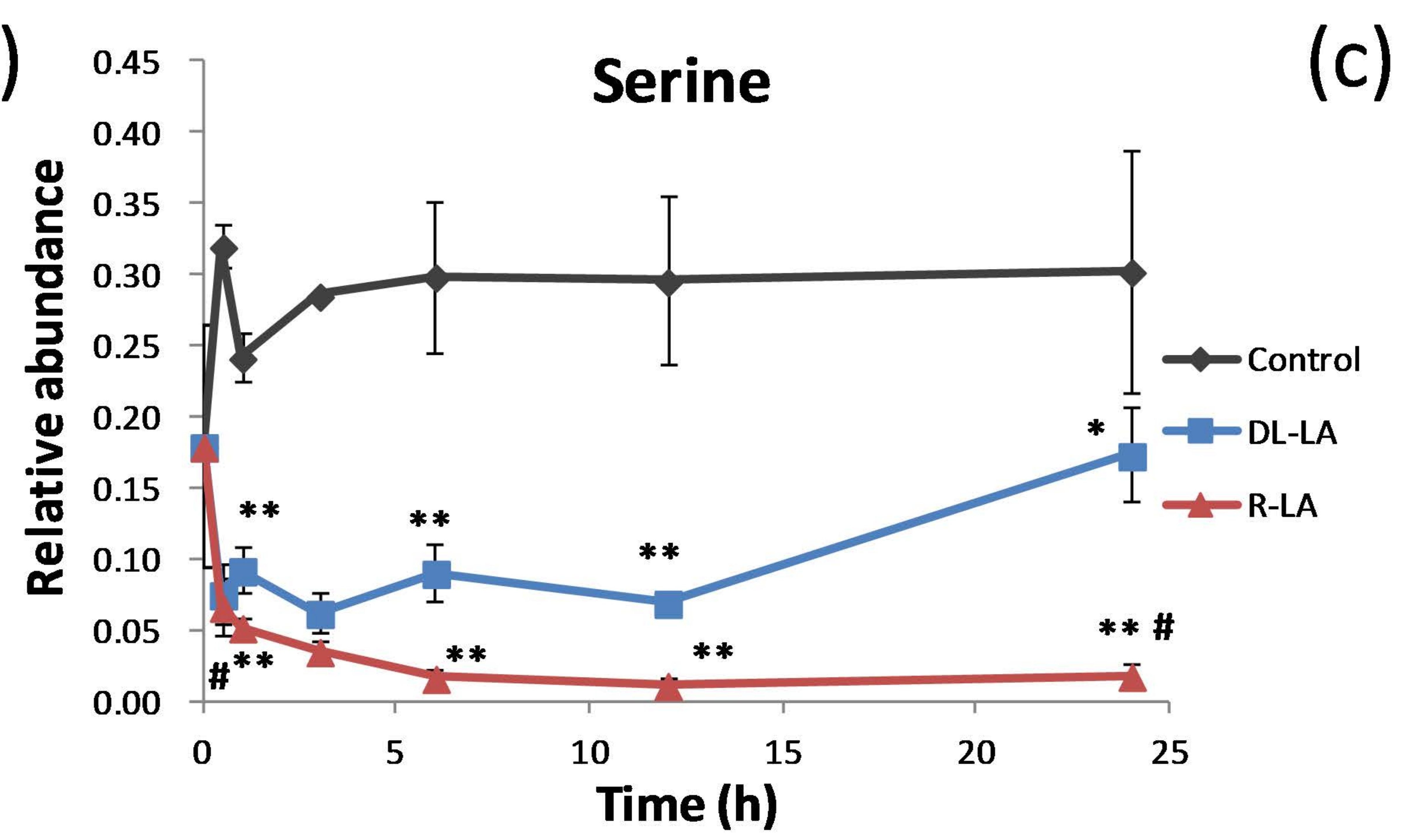

(e)

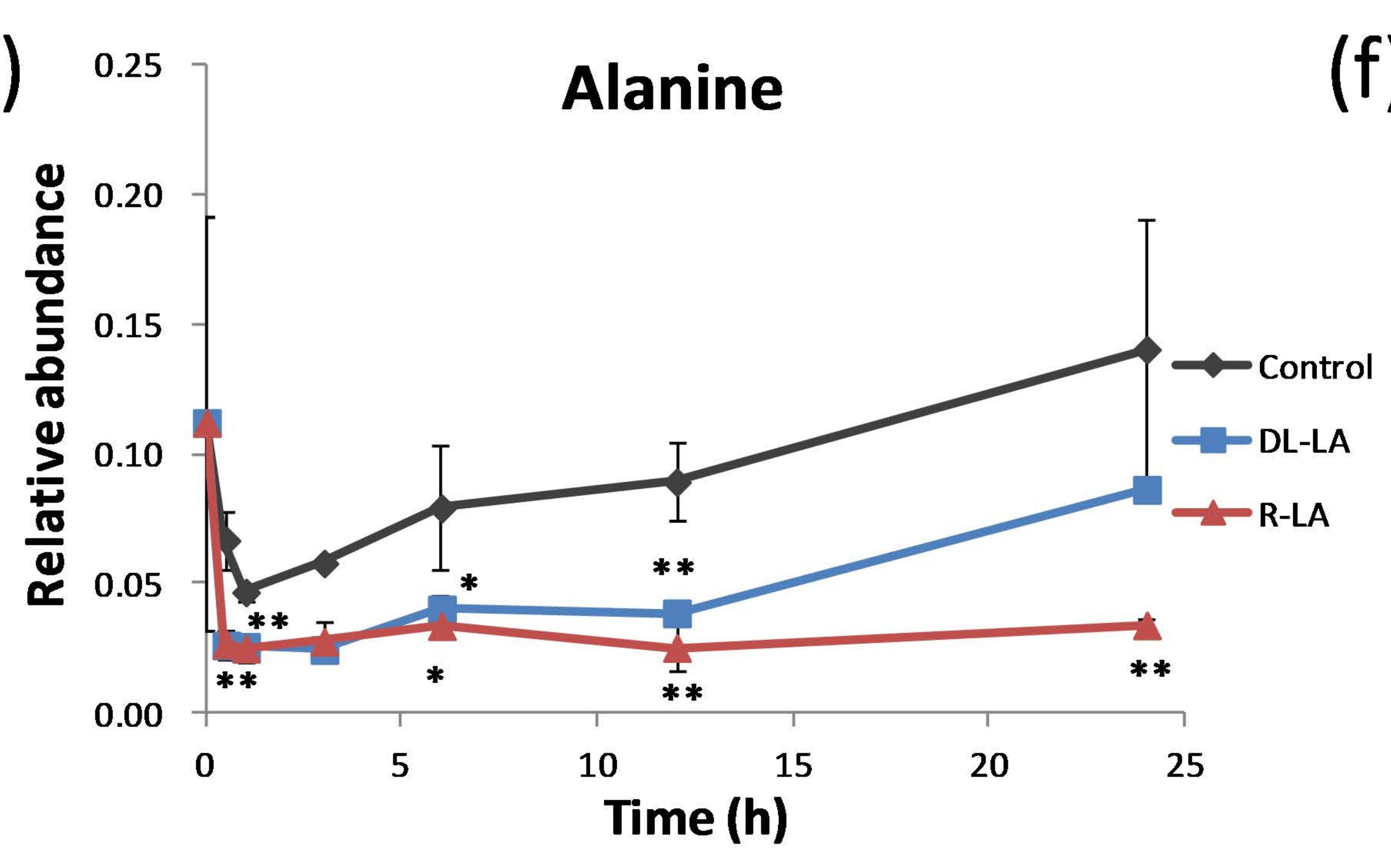

(h)

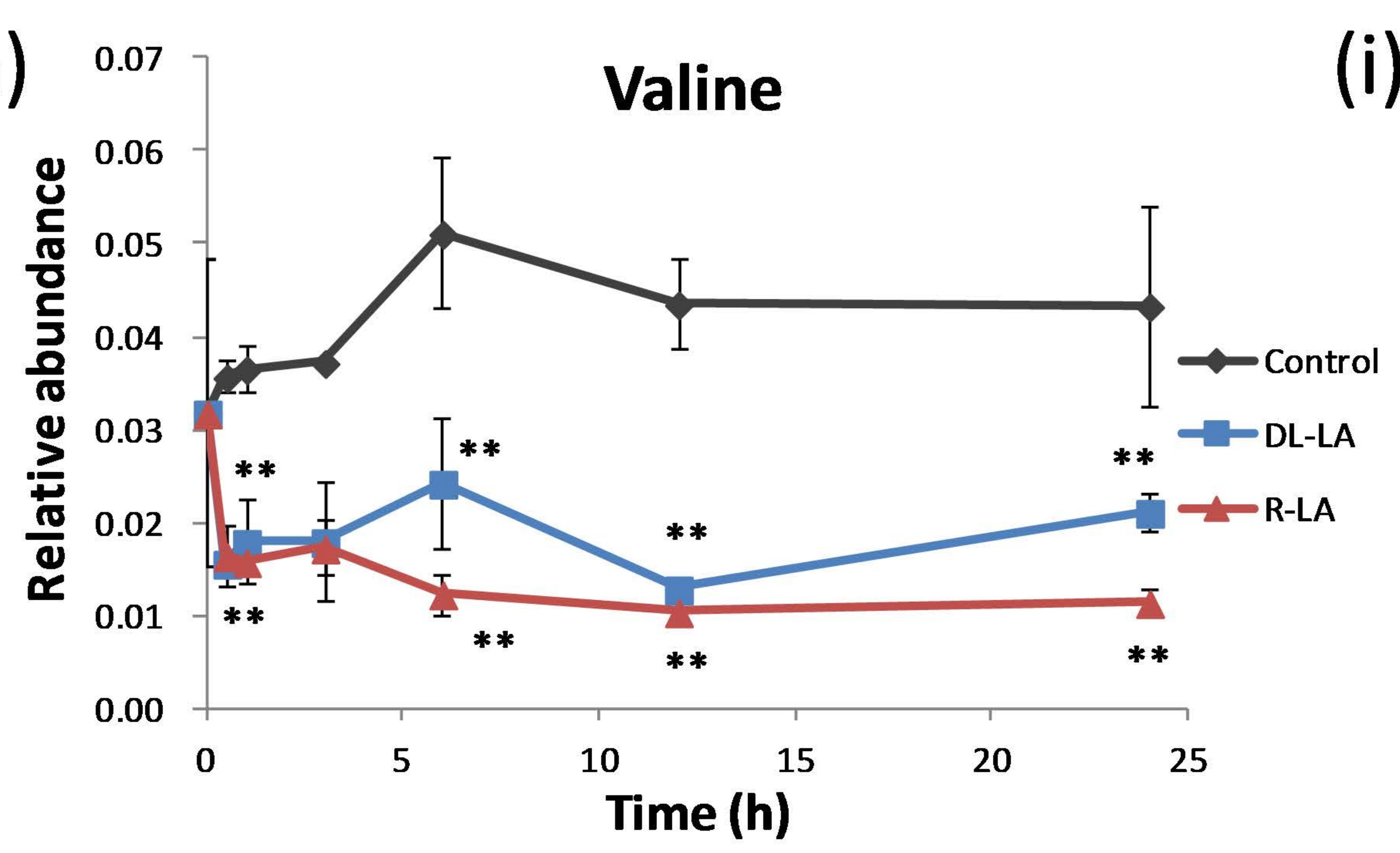

(k)

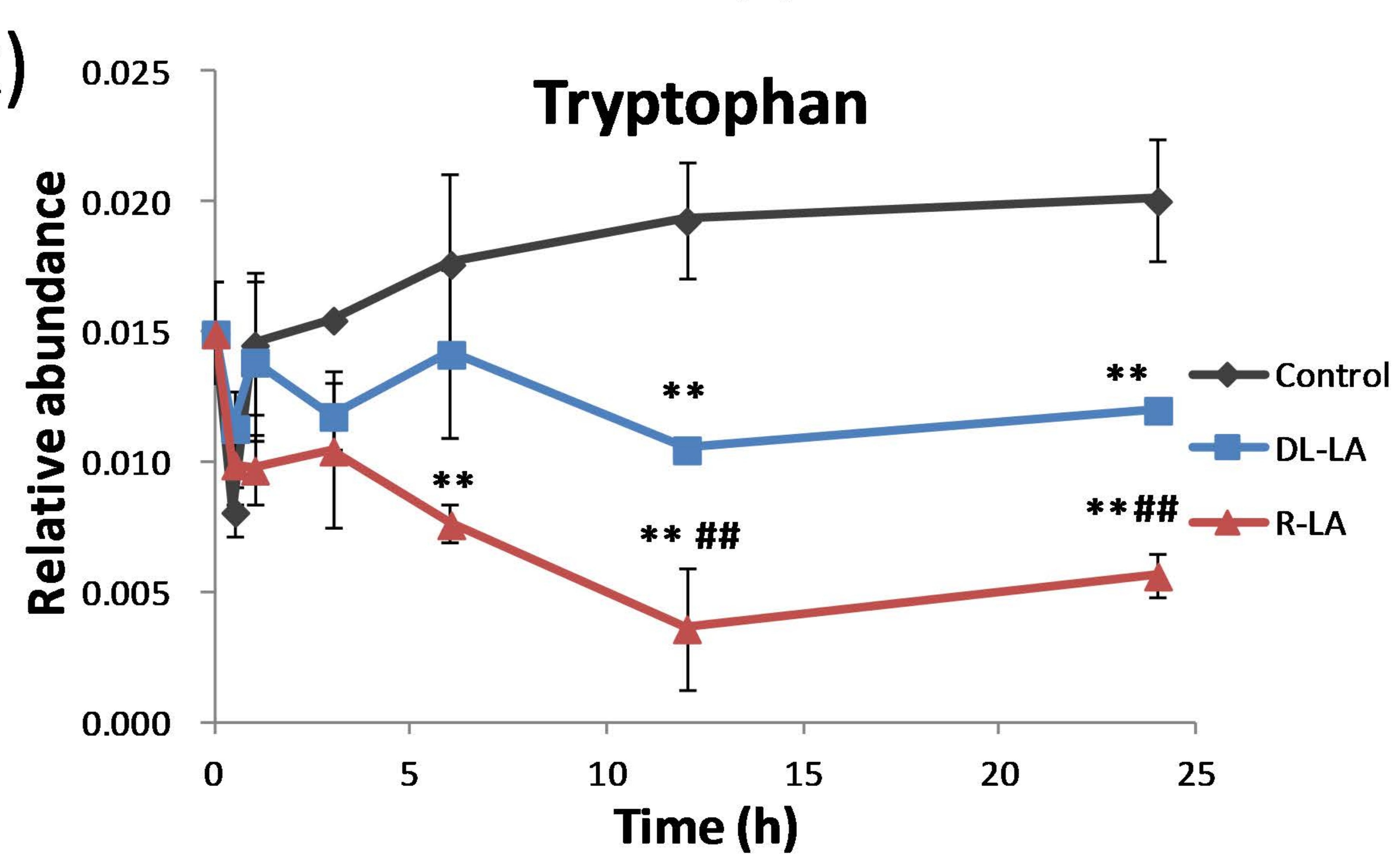

(m)

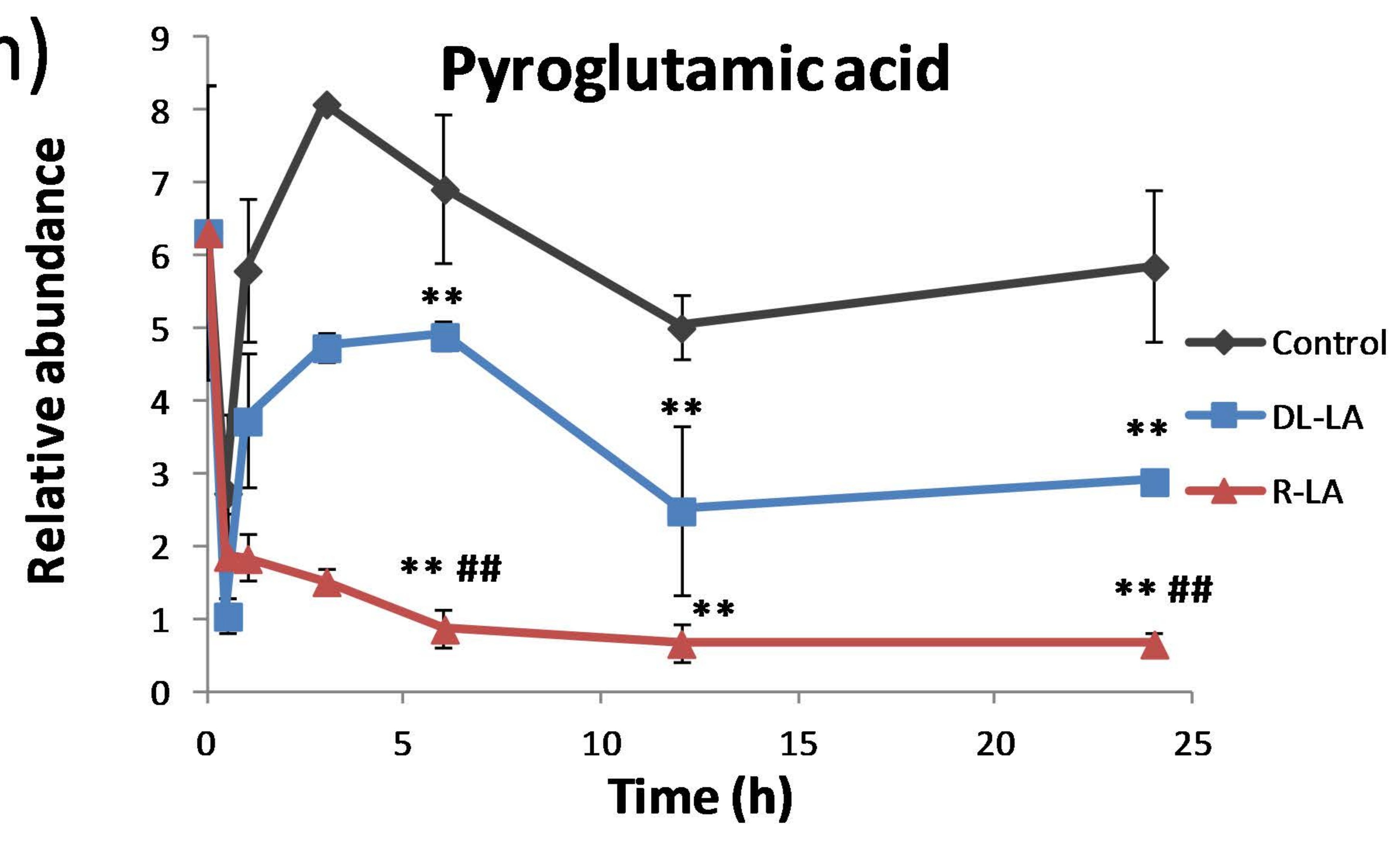

(c) $\left.{ }^{3.0}\right] \quad$ Glycine

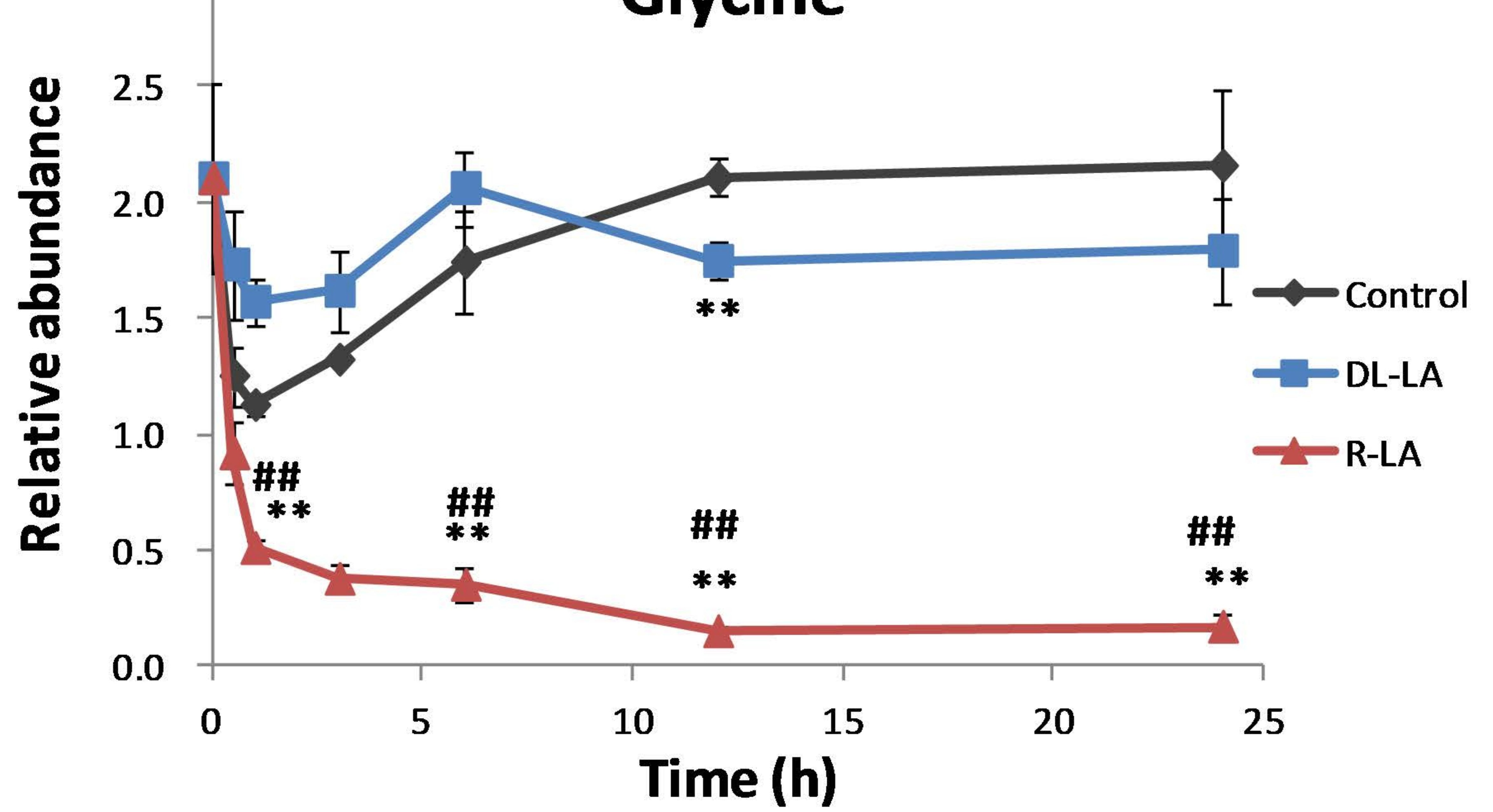

(f)

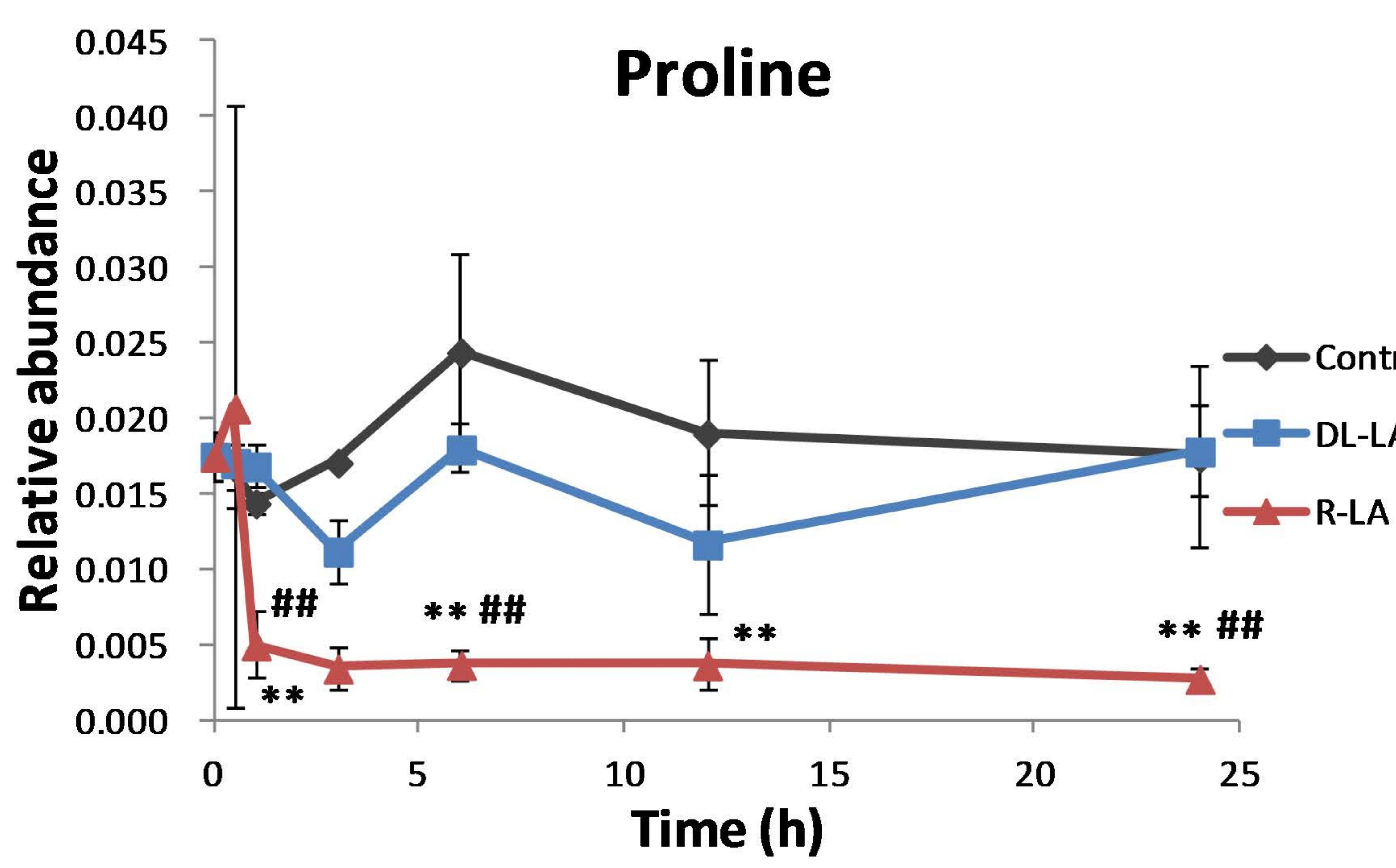

(i)

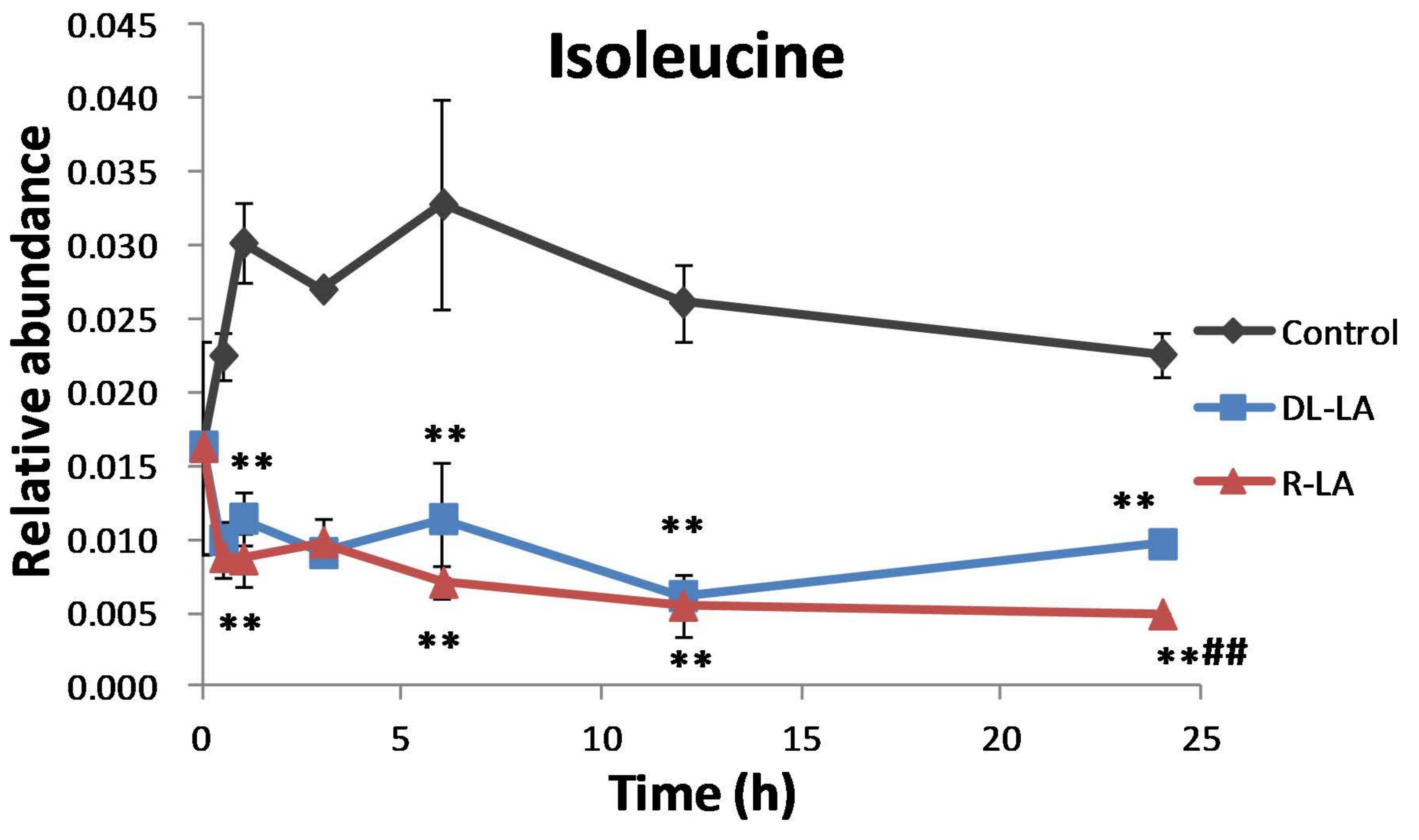


(a)

$0.5 \mathrm{~h}$ (PCA-X)

$\mathrm{t}$ [Comp. 1] t [Comp. 2]

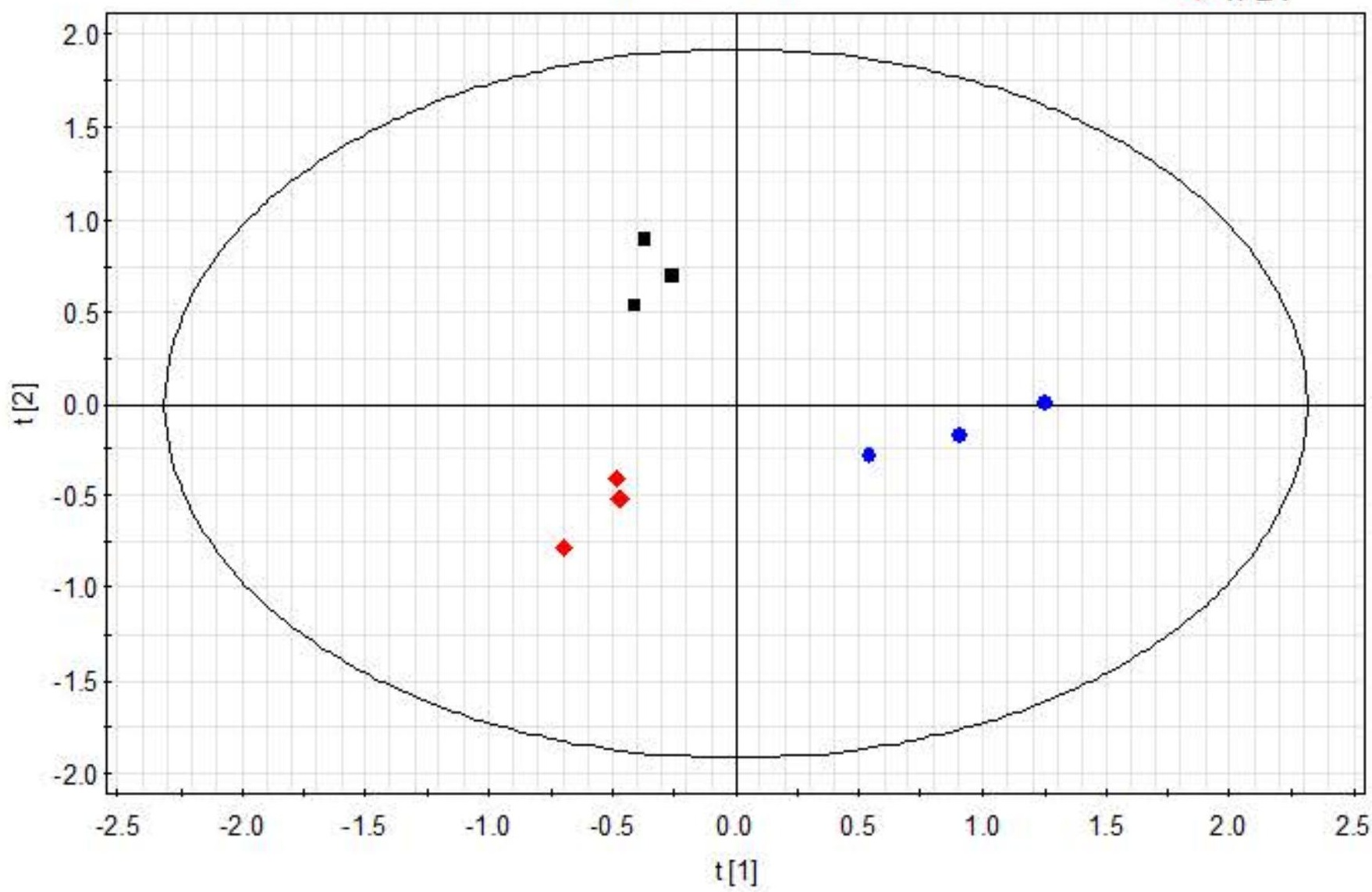

$\mathrm{R} 2 \mathrm{X}[1]=0.468931$

$\mathrm{R} 2 \mathrm{X}[2]=0.323229$

Ellipse: Hotelling T2 $(0.95)$

(b)

$6 h$ (PCA-X)

- Control

$\mathrm{t}$ [Comp. 1]/t [Comp. 2]

- DL-LA

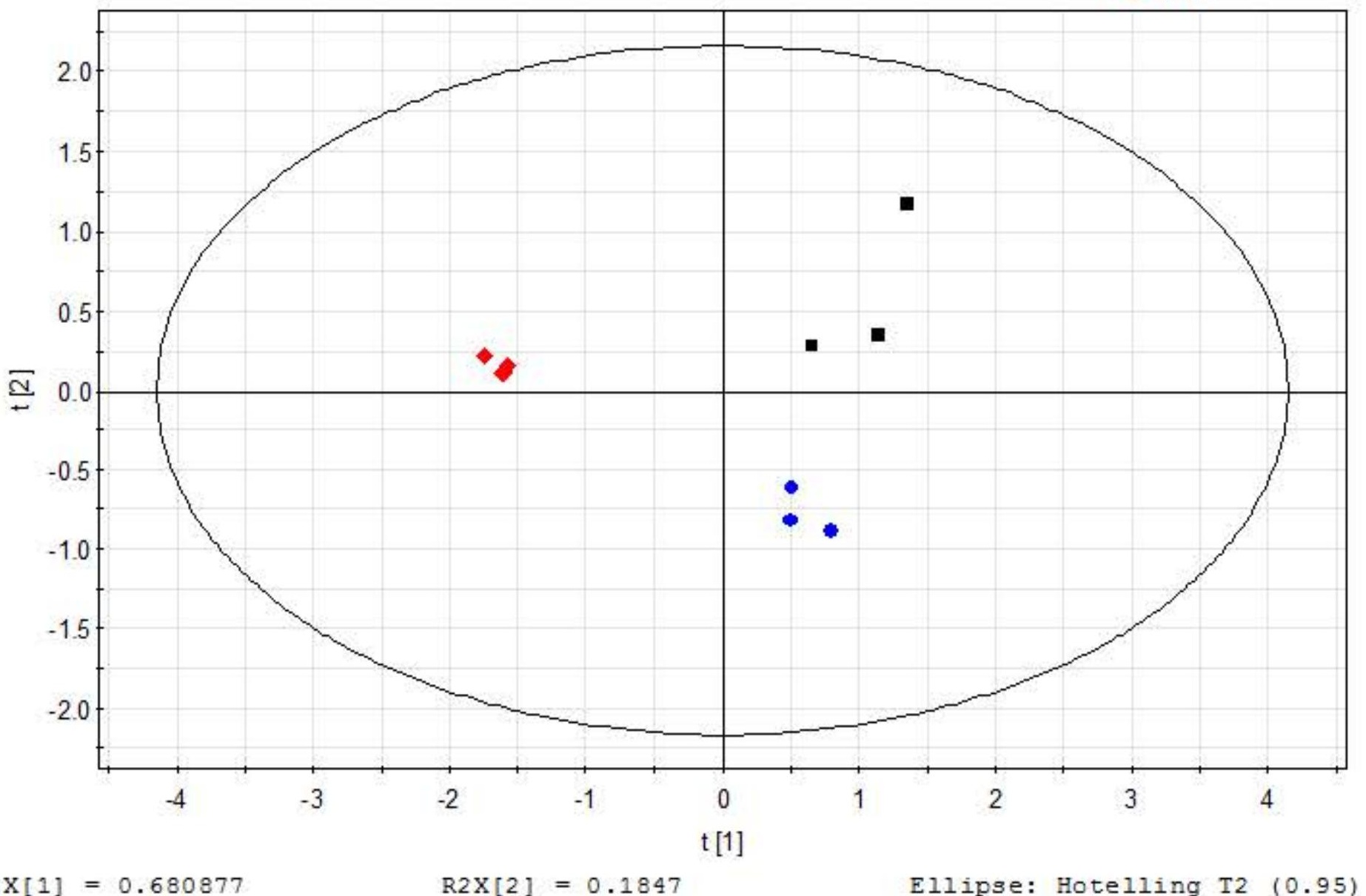

$R 2 X[1]=0.680877$

Ellipse: Hotelling T2 (0.95)

(c)

12h (PCA-X)

- Control

- DL-LA

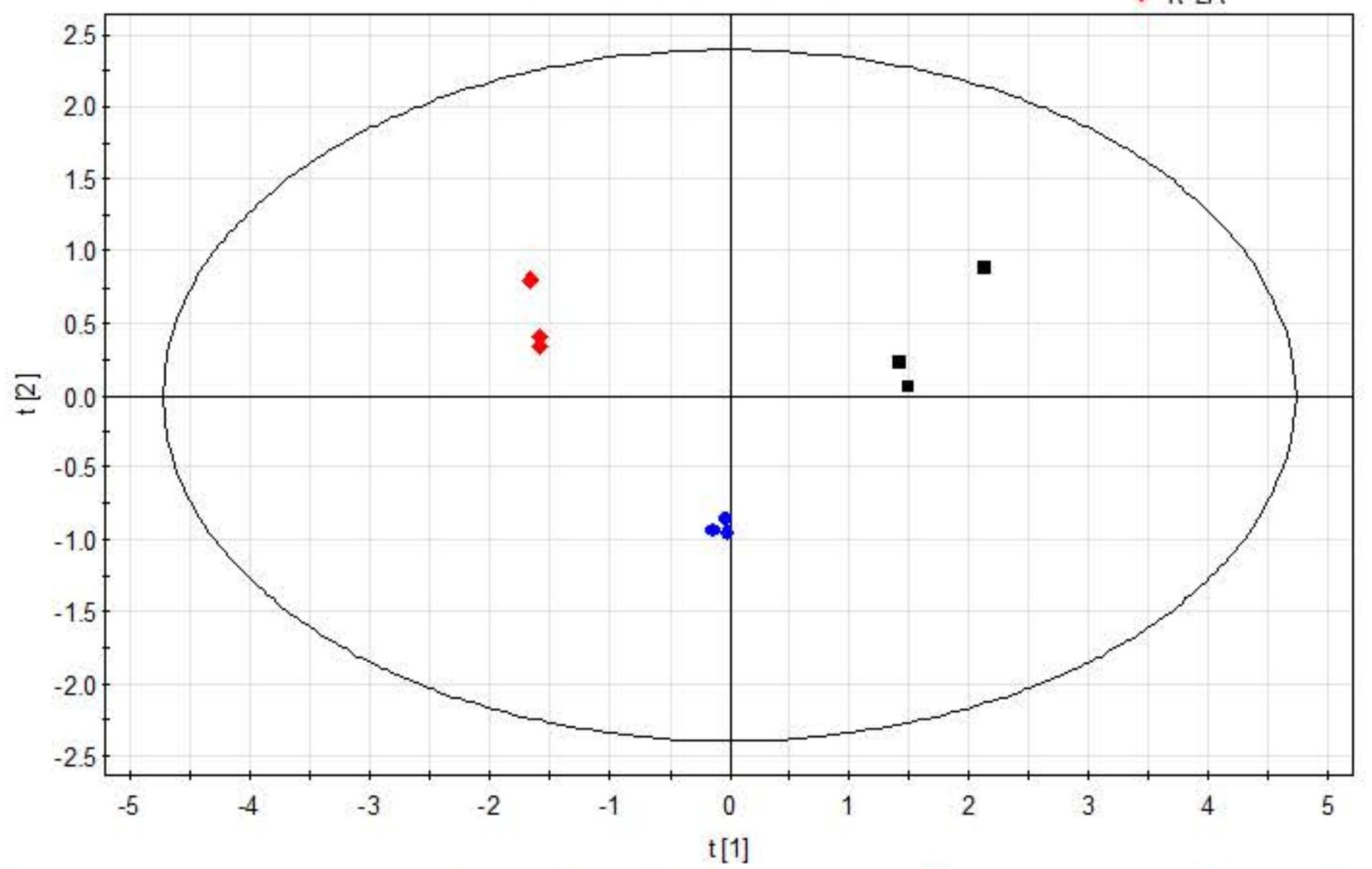


(a)

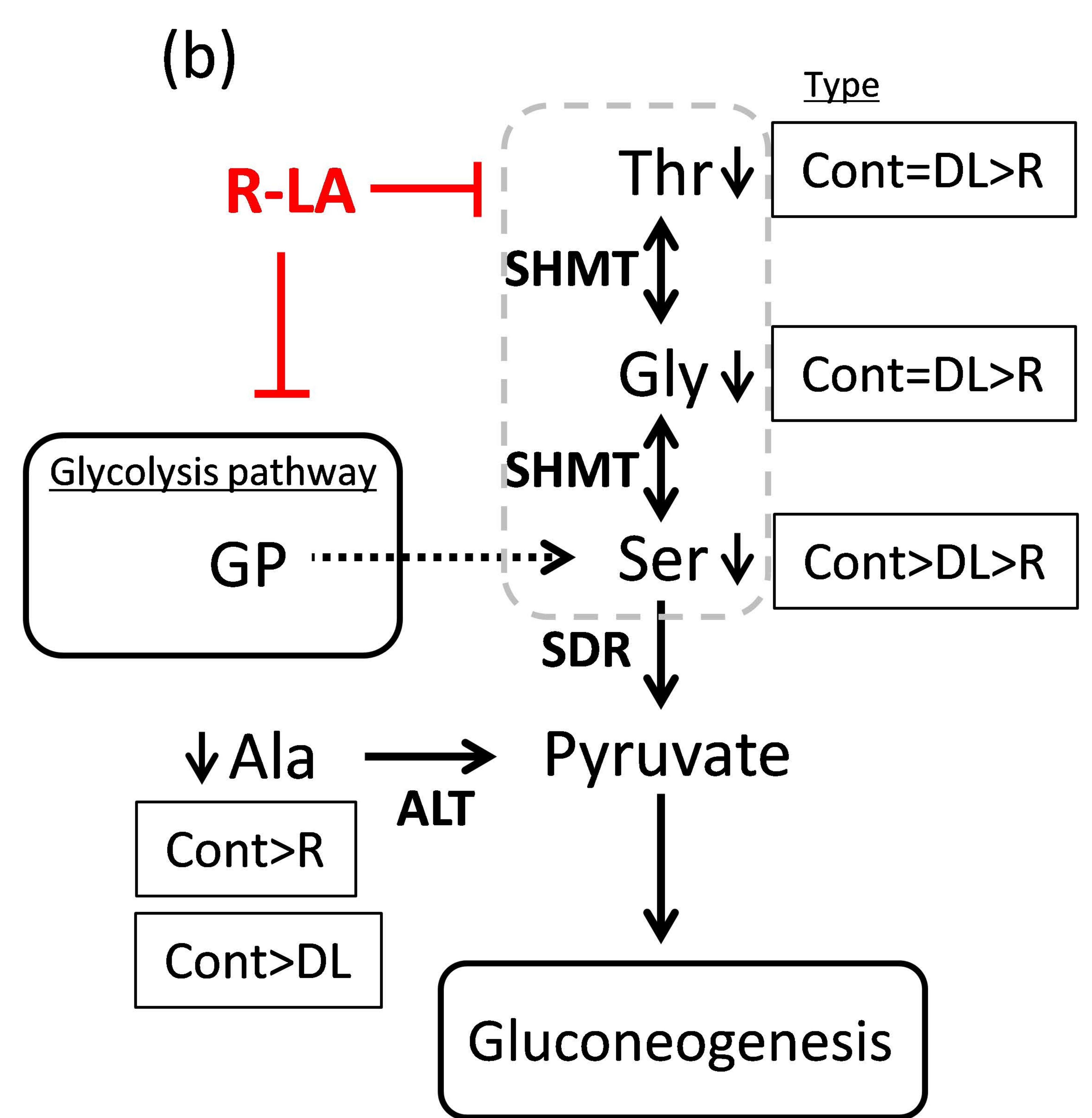

